\title{
Targeted delivery of celastrol to glomerular endothelium and podocytes for chronic kidney disease treatment
}

\author{
Qingsi Wu ${ }^{1,3}$, Jiading Wang ${ }^{2,3}$, Yuanfang Wang ${ }^{1,3}$, Ling Xiang ${ }^{1,3}$, Yulu Tan ${ }^{1,3}$, Jiaxing Feng ${ }^{1,3}$, Zhirong Zhang ${ }^{1,3}(\bowtie)$, \\ and Ling Zhang ${ }^{2,3}(\bowtie)$ \\ ${ }^{1}$ Key Laboratory of Drug Targeting and Drug Delivery Systems, Ministry of Education, West China School of Pharmacy, No.17, Block 3, Southern \\ Renmin Road, Chengdu 610041, China \\ ${ }^{2}$ College of Polymer Science and Engineering, Sichuan University, No.24, South Block 1, First Ring Road, Chengdu 610065, China \\ ${ }^{3}$ Med-X Center for Materials, Sichuan University, No.14 Section 3 South Renmin Road, Jinjiang District, Chengdu 610000, China
}

(C) Tsinghua University Press and Springer-Verlag GmbH Germany, part of Springer Nature 2021

Received: 13 July 2021 / Revised: 7 September 2021 / Accepted: 17 September 2021

\section{ABSTRACT}

The etiology of chronic kidney disease (CKD) is complex and diverse, which could be briefly categorized to glomerular- or tubularoriginated. However, the final outcomes of CKD are mainly glomerular sclerosis, endothelial dysfunction and injury, and chronic inflammation. Thus, targeted delivery of drugs to the glomeruli in order to ameliorate glomerular endothelial damage may help alleviate CKD and help enrich our knowledge. The herb tripterygium wilfordii shows therapeutic effect on kidney disease, and celastrol $(C L T)$ is one of its active ingredients but with strong toxicity. Therefore, based on the unique structure and pathological characteristics of the glomerulus, we designed a targeted delivery system named peptides coupled CLT-phospholipid lipid nanoparticles (PC-PLNs) to efficiently deliver CLT to damaged endothelial cells and podocytes in the glomerulus for CKD treatment and research. PC-PLNs could effectively inhibit inflammation, reduce endothelial damage, alleviate CKD severity, and reduce the toxicity of CLT. We also studied the mechanism of CLT in the treatment of nephropathy and found that CLT can increase the level of NO by increasing eNOS while inhibiting the expression of VCAM-1, thus provides an anti-inflammatory effect. Therefore, our study not only offered an efficient CKD drug formulation for further development, but also provided new medical knowledge about CKD.

\section{KEYWORDS}

celastrol, chronic kidney disease (CKD), glomerulus, endothelial cells, VCAM-1

\section{Introduction}

Chronic kidney disease $(\mathrm{CKD})$ refers to chronic kidney structure deterioration and dysfunction caused by various reasons. About $8 \%-16 \%$ of the global population suffer from CKD [1] and CKD prevalence may further increase in the next 10 years [2]. If not treated in time, CKD may develop into end-stage renal disease (ESRD). By then, patients can only rely on kidney transplantation and dialysis to maintain their lives, which brings dramatic drop of life quality and huge burden to health services [2]. Therefore, therapy to prevent CKD from progressing to ESRD is of great significance.

Celastrol (CLT) is an active ingredient extracted from the traditional Chinese medicine (TCM): tripterygium wilfordii. CLT has anti-inflammatory, anti-oxidant, immunosuppressive and antitumor effects [3-10]. As an ingredient of TCM, it displays significant therapeutic effects on various inflammatory diseases and autoimmune diseases $[4,5,7,10-16]$. In China, CLT has been widely used in the treatment of various kidney diseases. However, it also exhibits non-negligible toxicity $[7,11]$. Preliminary studies in our laboratory have shown that therapeutic doses of CLT have no significant impact on the renal function of rats, but can cause pathological changes in heart, liver, and brain [11]. In vivo distribution studies also show that CLT could not selectively distribute at kidney [11]. Therefore, to improve the efficiency of CLT and reduce its toxicity, a delivery system to selectively deliver CLT to the diseased kidney maybe highly valuable.

On the other hand, our knowledge about CKD etiology is still far from sufficient. CKD is generally progressive, regardless of the cause, its final pathological features include chronic inflammation, worsening interstitial fibrosis, glomerular sclerosis, and endothelial dysfunction $[17,18]$. In the kidney, the glomerulus filters the blood by its filter barrier and its pathological changes play an important role in CKD $[1,2,19]$. Hence, current CKD therapies with unclear mechanism, such as CLT, may actually alleviate glomerular damage. As known, because of its unique mechanical barrier and charge barrier, the glomerulus is selective to the permeability of substances and it is easier to accumulate particles with positive charge and of certain size range [11,20-23]. These characteristics could hence be exploited to design glomerulustargeting delivery systems $[11,22,24]$. Based on glomerulustargeting, drug delivery systems (DDS) that can increase drug accumulation in diseased cells may further improve drug efficiency and reduce toxicity to nearby tissues.

So far, most CKD studies have focused on podocyte damage, which may cause proteinuria, glomerulosclerosis, etc. [1, 2, 25-27]; while few studies have paid attention to endothelial damage. As 
the first line of direct contact with blood circulation, glomerular endothelial cells are extremely vulnerable to the attack of circulating substances such as blood glucose, blood lipids and inflammatory mediators. When endothelial cells are damaged, glycocalyx components on the surface may lose and the charge barrier could change, which may lead to proteinuria $[19,23,28]$. Meanwhile, the loss of glycocalyx may expose the adhesion molecules on the surface of endothelial cells, which will cause the adhesion and aggregation of leukocyte to endothelial cells, worsening inflammation, and further cause endothelial dysfunction $[19,23,28]$. When endothelial cells are stimulated by inflammation, the expression of VCAM-1 on the surface will be up-regulated $[18,29]$. It is reported that the linear peptide VHPKQHRGGSKGC can bind VCAM-1, but most studies focused on using this peptide for diagnosis and it has not yet been used to target glomerular endothelium as far as we know [30].

In this study, we thus fabricated peptides coupled CLTphospholipid lipid nanoparticles (PC-PLNs) with zeta potential of about $+12 \mathrm{mV}$ and particle size of about $110 \mathrm{~nm}$, aiming to deliver CLT to glomerular endothelial cells. We found that because inflammatory podocytes also highly express VCAM-1, the in vitro uptake of inflammatory endothelial cells and podocytes both has been significantly improved compared with normal cells. Considering that the podocytes also play an important role in $\mathrm{CKD}$, this delivery system may "kill two birds with one stone" [27,31]. Further experiments also showed that the modification of peptides both increased nanoparticles accumulation in inflammatory kidneys and enhanced their uptake by inflammatory endothelial cells and podocytes. In vivo assessments showed that PC-PLNs significantly ameliorated glomerular damage in immunoglobulin A nephropathy (IgAN) mice, which is likely related to alleviated endothelial damage. In mice with chronic progressive nephropathy induced by cisplatin, PC-PLNs treatment also displayed the strongest therapeutic effect among different treatments. These experiments verified that it is possible to treat $\mathrm{CKD}$ by targeting diseased glomeruli and treating glomerular damage. In addition, safety evaluation of PC-PLNs demonstrated that it significantly reduced the systemic side effects of CLT.

Using PC-PLNs, we also investigated the therapeutic mechanism of CLT in kidney. We found that levels of VCAM-1 and TNF- $\alpha$ in mice with nephropathy increased while the levels of CD34, eNOS, and NO decreased. After PC-PLNs treatment, the levels of VCAM-1 and TNF- $\alpha$ decreased, while the levels of CD34, eNOS and NO increased.

In conclusion, we successfully constructed a CLT-loaded delivery system, PC-PLNs. With dual active and passive targeting properties, PC-PLNs improved glomerular targeting to both endothelial cells and podocytes. At these sites, CLT may facilitate the repair of damaged cells in the glomerulus via upregulation of eNOS and downregulation of VCAM-1. In this way, PC-PLNs displayed strong amelioration of $\mathrm{CKD}$ progression and reduced CLT toxicity. These data also imply that at least in IgAN and chronic progressive nephropathy $(\mathrm{CPN})$ mice, glomerular damage is a major contributor to $\mathrm{CKD}$ progression and endothelial glomerular injury plays an important role.

\section{Experimental}

\subsection{Materials}

CLT was purchased from Chengdu Must Biotechnology (Chengdu, China); purified egg yolk lecithin (Lipoid E80) was obtained from LIPOID GMBH (Ludwigshafen, Germany); cholesterol (Chol) was purchased from Kelong Chemicals Co.,
Ltd. (Chengdu, China); (2,3-dioleoyloxy-propyl)trimethylammonium-chloride (DOTAP) and 1,2-distearoyl-sn-glycero-3-phosphoethanolamine-N-[methoxy(polyethylene glycol)2000] (DSPE-PEG2000) were purchased from A.V.T. (Shanghai, China) Pharmaceutical Tech Co., Ltd. (Shanghai, China); 1,2distearoyl-sn-glycero-3-phosphoethanolamine-N-[maleimide (polyethylene glycol)-2000] (DSPE-PEG2000-Mal) was purchased from Laysan Bio (USA); soybean oil was provided by Beiya Medical Oil (Tieling, China); peptide (VHPKQHRGGSKGC) was synthesized by China Peptides Co., Ltd. (Suzhou, China).

IFN- $\gamma$ was purchased from PeproTech (NJ, USA); lipopolysaccharides (LPS) was purchased from Beijing Solarbio Science \& Technology Co., Ltd. (Beijing, China); 1,19-dioctadecyl3,3,39,39-tetra-methylindodicarbocyanine (DiD) was purchased from Biotium (Hayward, USA); staphylococcus auresus enterooxin B (SEB) was purchased from Toxin Technology (USA); bovine serum albumin (Albumin Bovine V, BSA) was purchased from BioFroxx (Einhausen, Germany); cisdiammineplatinum dichloride was purchased from Shanghai Macklin Biochemical Co., Ltd. (Shanghai, China); cell counting kit8 (CCK-8) was purchased from APExBIO Technology LLC (Houston, TX, USA); rabbit anti-PODXL polyclonal antibody and rabbit anti-ESM1 polyclonal antibody were purchased from Biosynthesis Biotechnology Inc. (Beijing, China); anti-VCAM-1 and anti-VCAM1 antibody [MK-2] (FITC) were both purchased from Abcam (MA, USA); anti-eNOS antibody and anti-TNF- $\alpha$ were both purchased from Abcam (MA, USA); $\mathrm{NaN}_{3}$, chlorpromazine, dimethyl amiloride and nystatin were purchased from Sigma-Aldrich (USA). All other chemical reagents and solvents were of analytical grade or above.

\subsection{Animals and cell culture}

Healthy male Balb/c mice (6-8 weeks) were purchased from Dashuo Biotechnology (Chengdu, China) and raised under standard conditions. All animal experiments were carried out in accordance with the protocol of the National Act on the use of experimental animals (China) and were approved by the Institutional Animal Care and Ethic Committee of Sichuan University.

Mouse glomerulus endothelial cell (MRGEC), and podocyte (MPC5) cells were purchased from the China Center for Type Culture Collection. MRGEC was maintained in DMEM (Hyclone, UT, USA) with high-glucose supplemented with $10 \%(\mathrm{v} / \mathrm{v})$ fetal bovine serum (FBS, GIBCO, USA), streptomycin $\left(50 \mathrm{U} \cdot \mathrm{mL}^{-1}\right)$ and penicillin (50 U.mL $\mathrm{mL}^{-1}$ ) (Solarbio, Beijing, China). MPC5 was cultured at $33{ }^{\circ} \mathrm{C}$ for proliferation. Unless otherwise stated, 5 and 14 days before experiments, respectively, MPC5 was transferred to $37{ }^{\circ} \mathrm{C}$ for differentiation. And MPC5 was maintained in RPMI 1640 (Hyclone, UT, USA) with high-glucose supplemented with $10 \%(\mathrm{v} / \mathrm{v})$ FBS, streptomycin $\left(50 \mathrm{U} \cdot \mathrm{mL}^{-1}\right)$ and penicillin $\left(50 \mathrm{U} \cdot \mathrm{mL}^{-1}\right)$ (Solarbio, Beijing, China). All cells were incubated in a humidified atmosphere containing $5 \% \mathrm{CO}_{2}$ at $37^{\circ} \mathrm{C}$.

We used sample sizes of five for animal experiments, and of three for cell experiments. No data or animals were excluded due to being outliers.

\subsection{Preparation and characterization of PC-PLNs}

\subsubsection{Preparation PC-PLNs}

First, CLT-phospholipid lipid nanoparticles (C-PLNs) were prepared by a solvent evaporation method. Briefly, $1.00 \mathrm{mg}$ CLT powder, $14.28 \mathrm{mg}$ lipoid E80, $2.00 \mathrm{mg}$ Chol, $1.32 \mathrm{mg}$ DOTAP, $3.00 \mathrm{mg}$ DSPE-PEG2000 and $1.40 \mathrm{mg}$ DSPE-PEG2000-Mal were co-dissolved in $5 \mathrm{~mL}$ solvent (dichloromethane (DCM):ethyl acrylate (EA): $\left.\mathrm{CHCl}_{3}=57: 110: 59\right)$ under gently stirring at room 
temperature for $1 \mathrm{~h}$. Then, the organic solvent was removed by rotary evaporation under reduced pressure to form CLTphospholipid complex (C-PC). Then $3 \mu \mathrm{L}$ soybean oil and $3 \mathrm{~mL}$ solvent (DCM:EA: $\mathrm{CHCl}_{3}=$ 57:110:59) were added into C-PC, under gently stirring at room temperature for $10 \mathrm{~min}$. After that, the organic solvent was removed by rotary evaporation again, and the film was hydrated in $4 \mathrm{~mL} 0.02 \mathrm{M}$ hepes buffer. Next, above formulation was sonicated about $7 \mathrm{~min}$ in a sonicator $(210 \mathrm{~W})$ to obtain the C-PLNs.

PC-PLNs were prepared by mixing peptides with C-PLNs at $37{ }^{\circ} \mathrm{C}$ for $4 \mathrm{~h}$ stirring. Unconjugated peptides were removed by dialysis against distilled water at room temperature for $24 \mathrm{~h}$.

\subsubsection{Characterization of PC-PLNs}

Particle size distribution and zeta potential of PC-PLNs were determined by dynamic light scattering with a Zetasizer Nano ZS90 instrument (Malvern, UK). The morphology of PC-PLNs was observed by transmission electron microscopy (TEM, Field Electron and Ion Company, Oregon, USA) with 2\% phosphotungstic acid staining. Encapsulation efficiency (EE\%) of CLT was measured using high performance liquid chromatography (HPLC). The following formula was used: EE\% $=($ mass of loaded CLT/total amount of CLT $) \times 100 \%$. The drug loading rate $(\mathrm{DL} \%)$ was calculated by the formula: DL\% $=[$ mass of loaded CLT/(mass of loaded CLT + total mass of materials) $] \times$ $100 \%$.

\subsection{Cellular uptake of different potential DiD-loaded PLNs (D-PLNs) in vitro}

MRGEC and MPC5 cells were both seeded in 12-well plates of 1.5 $\times 10^{5}$ per well, cultured in medium with $10 \%$ FBS for $24 \mathrm{~h}$. Then, the medium was removed and $1 \mathrm{~mL}$ fresh serum-free medium containing different potential D-PLNs $\left(0.3 \mu \mathrm{g} \cdot \mathrm{mL}^{-1} \mathrm{DiD}\right)$ was added, and cells were incubated for another $4 \mathrm{~h}$. Finally, cells were harvested, washed three times with phosphate-buffered saline (PBS), and mean fluorescence intensity (MFI) was analyzed by flow cytometry (BD FACS Celesta, USA).

\subsection{Distribution of different potential D-PLNs in vivo}

Healthy male balb/c mice were randomly divided into four groups $(n=3)$ to receive Neg-D-PLNs, Neu-D-PLNs, Pos-D-PLNs or HPos-D-PLNs (100 $\left.\mu \mathrm{g} \cdot \mathrm{kg}^{-1} \mathrm{DiD}\right)$ via tail vein injection. Control mouse was injected with normal saline. After $2 \mathrm{~h}$, the mice were sacrificed and kidneys were imaged using a Caliper IVIS Lumina III system (PerkinElmer, USA).

\subsection{Cellular localization of different potential D-PLNs in kidney}

Healthy male balb/c mice were randomly divided into four groups $(n=3)$ to receive Neg-D-PLNs, Neu-D-PLNs, Pos-D-PLNs, HPosD-PLNs $\left(100 \mu \mathrm{g} \cdot \mathrm{kg}^{-1} \mathrm{DiD}\right)$ via tail vein injection. After $2 \mathrm{~h}$, the mice were sacrificed. The kidneys were firstly fixed in $4 \%$ paraformaldehyde fix solution, and then further dehydrated using $15 \%(\mathrm{w} / \mathrm{v})$ sucrose, then $30 \%(\mathrm{w} / \mathrm{v})$ sucrose. After that, they were snap-frozen in optimal cutting temperature compound (OCT) and sliced to $5-\mu \mathrm{m}$ sections, then $5 \%$ donkey serum blocking solution was added and the sections were incubated at room temperature for $30 \mathrm{~min}$. Then, the first group was stained with rabbit anti-ESM1 polyclonal antibody goat (1:100; bioss, bs3615R), followed by FITC conjugated mouse anti-rabbit antibody (1:100; bioss, bs-0295M-FITC). The second group was stained with rabbit anti-PODXL polyclonal antibody (1:100; bioss, bs1345R), followed by FITC conjugated mouse anti-rabbit antibody (1:100; bioss, bs-0295M-FITC). Finally, the stained sections were obtained by using a confocal laser scanning microscope (CLSM, LSM 800, Zeiss, Jena, Germany).

\subsection{Cellular uptake of D-PLNs by LPS-MRGEC and LPS- MPC5}

MRGEC and MPC5 cells were both seeded in 12-well plates of 1.5 $\times 10^{5}$ per well, cultured in medium with $1 \mu \mathrm{g} \cdot \mathrm{mL}^{-1}$ LPS for $24 \mathrm{~h}$. Then, the medium was removed and $1 \mathrm{~mL}$ fresh serum-free medium containing different D-PLNs $\left(0.3 \mu \mathrm{g} \cdot \mathrm{mL}^{-1} \mathrm{DiD}\right)$ was added, and cells were incubated for another $4 \mathrm{~h}$. Finally, the cells were harvested and washed three times with PBS, and MFI was analyzed by flow cytometry (BD FACS Celesta, USA).

\subsection{VCAM-1 expression on LPS-MRGEC and LPS- MPC5}

MRGEC and MPC5 cells were both seeded in 12-well plates of 1.5 $\times 10^{5}$ per well, cultured in medium with or without $1 \mu \mathrm{g} \cdot \mathrm{mL}^{-1} \mathrm{LPS}$ for $24 \mathrm{~h}$. Then, the cells were harvested and washed once with PBS. Each tube of cells was blocked by adding $50 \mu \mathrm{L}$ of PBS solution containing $2 \%$ FBS, and followed by $50 \mu \mathrm{L}$ of FITClabeled anti-VCAM-1 antibody (1:100; abcam, ab24853). After incubating at $4{ }^{\circ} \mathrm{C}$ for $40 \mathrm{~min}$, the cells were centrifuged to discard the supernatant and washed three times with PBS. Finally $350 \mu \mathrm{L}$ of PBS was added for resuspension. The parent of VCAM-1 positive cells was determined by flow cytometry (BD FACS Celesta, USA).

\subsection{Competitive inhibition assay}

MRGEC and MPC5 cells were both seeded in 12-well plates of 1.5 $\times 10^{5}$ per well, cultured in medium with $1 \mu \mathrm{g} \cdot \mathrm{mL}^{-1} \mathrm{LPS}$ for $24 \mathrm{~h}$. Then, the medium was removed. Cells were pre-incubated with or without anti-VCAM1 antibody (1:40, Abcam, ab134047) for $30 \mathrm{~min}$ before treatment with peptides coupled D-PLNs $(0.3$ $\left.\mu \mathrm{g} \cdot \mathrm{mL}^{-1} \mathrm{DiD}\right)$ for $4 \mathrm{~h}$. Then, the cells were harvested and washed three times with PBS, and MFI was analyzed by flow cytometry (BD FACS Celesta, USA).

\subsection{Uptake inhibition study of D-PLNs in LPS-MRGEC and LPS-MPC5}

To explore potential pathway(s) of D-PLNs, cellular uptake was measured at $4{ }^{\circ} \mathrm{C}$, as well as after $1 \mathrm{~h}$ pre-treated with the following inhibitors at $37{ }^{\circ} \mathrm{C}$ : sodium azide $(154 \mu \mathrm{M})$, chlorpromazine $(25 \mu \mathrm{M})$, dimethyl amiloride $(100 \mu \mathrm{M})$, or nystatin $(25 \mu \mathrm{M})$. Then the cells were further incubated with DPLNs for $4 \mathrm{~h}$. Finally, cells were collected and analyzed as above.

\subsection{Cell viability}

Cell viability was examined using the CCK-8 (APExBIO, Houston, TX, USA). Cells were seeded in 96-well plates of $1 \times 10^{4}$ cells per well. Next day, cells were treated for another $24 \mathrm{~h}$ with free CLT, negative charged CLT-loaded PLNs (NC-PLNs), CPLNs or PC-PLNs. Then $10 \mu \mathrm{L}$ of CCK-8 solution was added to each well and plates were incubated for $2 \mathrm{~h}$. The absorbance was measured at $450 \mathrm{~nm}$ using SPARK 10M (Tecan, Austria). Blank wells (culture media and CCK-8) and control wells (untreated cells, culture media and CCK-8) were also detected.

\subsection{IgAN model}

The immunoglobulin A (IgA) nephritis model induced by heterologous protein is an ideal model for studying IgAN. Based on the previous method used by our research group [22], the mouse model was induced by "BSA + SEB + LPS": BSA was dissolved in $8.5 \mathrm{mM}$ hydrochloric acid and mice were 
administrated intragastrically at a dose of $200 \mathrm{mg} \cdot \mathrm{kg}^{-1}$ every other day for 5 weeks; then mice were injected with $20 \mathrm{mg} \cdot \mathrm{kg}^{-1} \mathrm{BSA}$ daily for 3 consecutive days vis tail intravenous; finally, LPS and SEB mixed with sterile physiological saline solution were injected weekly into the tail vein at the doses of $0.5 \mathrm{mg} \cdot \mathrm{kg}^{-1}$ LPS and $0.05 \mathrm{mg} \cdot \mathrm{kg}^{-1} \mathrm{SEB}$ for 4 weeks. Several mice were sacrificed randomly to check whether the model was successful.

\subsection{Biodistribution of D-PLNs in nephropathy mice}

In this experiment, we used CPN mice for test. CPN mice were randomly divided into four groups $(n=3)$ to receive free $\mathrm{DiD}$, ND-PLNs, D-PLNs or PD-PLNs $\left(100 \mu \mathrm{g} \cdot \mathrm{kg}^{-1} \mathrm{DiD}\right)$ via tail vein injection. Control mice were injected with normal saline. Meanwhile, healthy mice were treated as same. After $2 \mathrm{~h}$, the mice were sacrificed and kidneys were imaged using a Caliper IVIS Lumina III system (PerkinElmer, USA).

\subsection{Therapeutic efficacy evaluation in vivo}

Whether it was IgAN model or CPN model, the following therapeutic procedure was administered. A total of 25 model mice were randomized into five groups ( $n=5$ per group): blank, CLT, NC-PLNs, C-PLNs, or PC-PLNs. Except blank group, other each group was administrated at dose of $1 \mathrm{mg} \cdot \mathrm{kg}^{-1} \mathrm{CLT}$. Treatment was given on days $2,4,6$, and 8 after induction of kidney disease for once a day. The blank group and untreated healthy mice (NT group, $n=5$ ) were injected with an equivalent volume of saline as controls. After the treatment, each group was raised in a special metabolic cage for mice for one day and urine was collected for $24 \mathrm{~h}$. Finally, mice were sacrificed, blood and tissues were collected.

\subsection{Proteinuria measurement}

The urine was centrifuged twice at 3,000 rpm for $3 \mathrm{~min}$ to obtain the supernatant. Then urinary protein concentration was measured by using the Bradford Protein Assay Kit (Solarbio, Beijing, China).

\subsection{Renal function evaluation}

The collected blood was stood still for $30 \mathrm{~min}$ at $37^{\circ} \mathrm{C}$, then centrifuged at $6,000 \mathrm{rpm}$ for $8 \mathrm{~min}$. The levels of blood urea nitrogen (BUN) and creatinine (CREA) of the obtained serum were assayed on a Roche cobas c 311 automatic biochemical analyzer (Roche, Swit).

\subsection{Renal NO measurement}

The kidney tissue homogenate was extracted and the protein content was determined. Then, the NO concentration was determined according to the NO determination kit (built in Nanjing). The renal NO level is the ratio of NO concentration to protein content.

\subsection{Renal glomerular histopathology}

After sacrificing the mice, tissues were put in $4 \%$ paraformaldehyde at $37{ }^{\circ} \mathrm{C}$ for two days. Then $5-\mu \mathrm{m}$ paraffin sections were stained with hematoxylin-eosin (H\&E) and visualized by a light microscope (Olympus BX53, Tokyo, Japan). All analyses were performed by a qualified pathologist.

\subsection{Immunohistochemistry}

The 5- $\mu \mathrm{m}$ paraffin sections were dewaxed and rehydrated. Then peroxidase blocking buffer (DAKO Company) was used to block the endogenous peroxidase activity. After washing buffer DAKO, the sections were incubated at $37{ }^{\circ} \mathrm{C}$ with primary antibodies against one of the following antigens for $45 \mathrm{~min}$ : rabbit anti-
VCAM-1 polyclonal antibody, rabbit anti-CD34 polyclonal antibody, mouse eNOS antibody, goat anti-mouse IgA $\alpha$ chain (HRP) and anti-TNF- $\alpha$ antibody. As for the control groups, the primary antibody was replaced with buffer. After adding $100 \mu \mathrm{L}$ Agent A (horseradish peroxidase-conjugated chemmate envision reagent), diaminobenzidine (DAB) was used for color reaction. Finally, each section was counterstained with hematoxylin. Finally, sections were photographed, semi-quantitative scores [32] and quantitative integrated optical density (IOD) values were determined for the staining by one professional pathologist.

\subsection{Safety evaluation}

Fifteen healthy balb/c mice were randomized into three groups ( $n=5$ per group): blank, CLT or PC-PLNs. The blank group received saline; the CLT group treated with free CLT and the PCPLNs group treated with PC-PLNs (both are at a CLT dose of $\left.1 \mathrm{mg} \cdot \mathrm{kg}^{-1}\right)$. Saline or drugs were administered to mice via tail vein injection once every day for a total of four injections. On day 8 , mice were sacrificed, blood and tissues were collected. Tissues were washed, fixed, and stained with $\mathrm{H} \& \mathrm{E}$, histological lesions were observed and photographed under a light microscope (Olympus BX53, Tokyo, Japan). The levels of alanine aminotransferase (ALT), aspartate aminotransferase (AST), CREA, BUN, creatine kinase isoenzymes (CKMB) and lactic dehydrogenase (LDH) of the obtained serum were assayed on a roche cobas c 311 automatic biochemical analyzer (Roche, Swit).

\subsection{Statistical analysis}

Results are measured as mean \pm standard deviation. Statistical analysis was produced by Graphpad Prism 8.0 (GraphPad Software, CA, USA). Inter-group differences were analyzed by student's $t$-test or one-way analysis of variance (ANOVA) when multiple groups were compared. Differences were considered statistically significant when $p<0.05$.

\section{Results and discussion}

\subsection{Fabrication and optimization of PLNs and PC-PLNs}

As introduced, charge and size are essential for glomerulus accumulation. However, the exact surface charge of nanoparticle needs fine control for optimal targeting effect, as it is well known high positive charge $(>15 \mathrm{mV}$ ) could lead to fast protein corona formation and strong toxicity; meanwhile, nanoparticles would be quickly cleared out of the body $[33,34]$. Therefore, controlling the charge within $15 \mathrm{mV}$ can help the nanoparticles better accumulate in the kidney and be as non-toxic to the body as possible $[21,35]$.

In this study, phospholipid lipid nanoparticles (PLNs) were first generated with different surface charges by controlling the amount of cationic phospholipid materials used (Section S1.3 in the Electronic Supplementary Material (ESM)). D-PLNs with different potentials were generated as following: Neg-D-PLNs (-13.33 \pm $0.80 \mathrm{mV})$; Neu-D-PLNs $(-1.00 \pm 0.36 \mathrm{mV})$; Pos-D-PLNs $(6.20 \pm$ $0.32 \mathrm{mV}$ ); HPos-D-PLNs $(11.60 \pm 0.92 \mathrm{mV}$ ) (Table S1 in the ESM). As nanoparticles with strong positive charge are highly toxic and tend to strongly accumulate in lung rather than kidney, we did not generate PLNs with $>12 \mathrm{mV}$ surface charge [33-36]. Then, the cellular uptake of these PLNs was investigated in vitro. Cell flow cytometry results showed that both MRGEC and MPC5 had the highest uptake of HPos-D-PLNs (Figs. 1(a) and 1(b)). Next, these PLNs were intravenously injected into mice and their kidneys were removed $2 \mathrm{~h}$ later to be imaged ex vivo. From these images, HPos-D-PLNs showed significantly higher accumulation than the other three treatment groups (Figs. 1(c) and 1(d)). To better understand the intrarenal accumulation of these PLNs, 
(a)

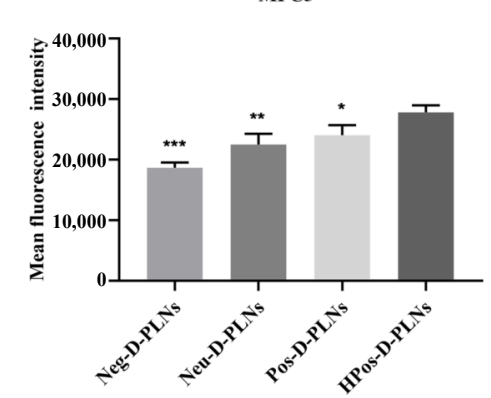

(c)

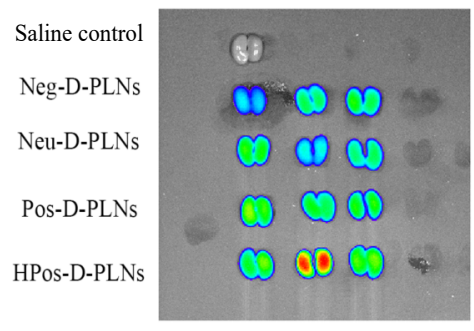

(b)

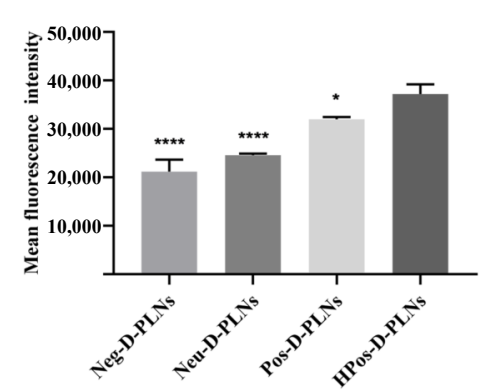

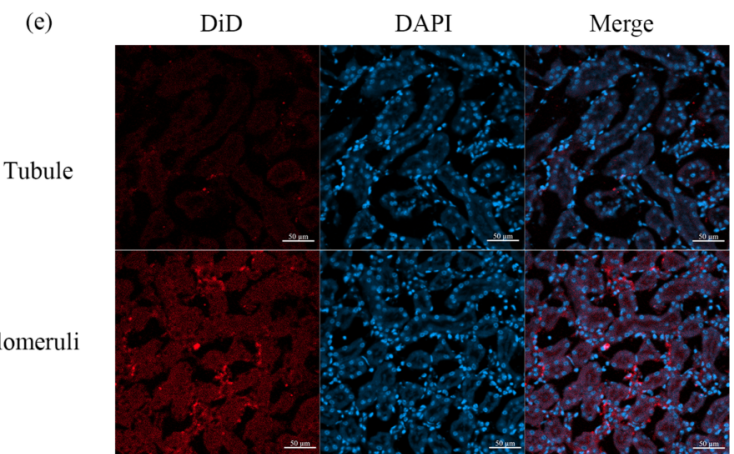

(d)

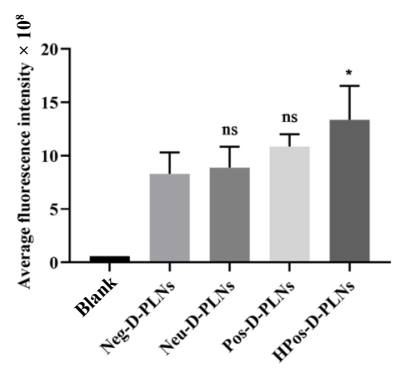

Figure 1 PLNs zeta-dependently accumulate in kidney. (a) and (b) Flow cytometric analyses of uptake of different zeta potentials D-PLNs by MPC5 cells (a) and MRGEC cells (b). All results are shown as mean $\pm \mathrm{SD}, n=3 .{ }^{\star} P<0.05,{ }^{* *} P<0.01$, ${ }^{* *} P<0.001{ }^{* * * *} P<0.0001$ vs. HPos-D-PLNs group. (c) In vivo fluorescence images of kidneys excised at $2 \mathrm{~h}$ after tail vein injection of D-PLNs. (d) Semiquantitative fluorescence intensity of excised kidneys in (c). All results are shown as mean \pm SD, $n$ $=3 .{ }^{\star} P<0.05$, ns, not significant vs. Neg-D-PLNs group. (e) Representative confocal images of D-PLNs localization at kidney, $n=3$. Scale bars, $50 \mu \mathrm{m}$.

kidneys were then sectioned and processed for further fluorescent imaging. Resulting images showed that D-PLNs mainly distributed in glomeruli, while only weak autofluorescence was detected in the tubular region (Fig. 1(e)). ESM1 and PODXL labelling showed that although all PLNs accumulated in the cells of the glomerulus, HPos-D-PLNs accumulation was the highest. Hence, the charge of HPos-D-PLNs appeared to be optimal for glomerular endothelium and podocytes targeting (Figs. 2(a) and 2(b)).

Based on above PLNs, we designed a positively charged drug delivery system (DDS) for glomerulus delivery. VCAM-1 is reported to be highly expressed on glomerular cell surface under inflammatory conditions $[18,29,37,38]$. Indeed, we confirmed that the expression of VCAM-1 did significantly increase on the surface of MRGEC (glomerular endothelial cell) and MPC5 (podocytes) cells after LPS treatment (which induces inflammation) (Figs. 3(a) and 3(b)). In order to further increase delivery efficiency to target cells, VCAM-1 binding peptide VHPKQHRGGSKGC was selected for PLNs modification $[39,40]$. CLT was first loaded into PLNs and then the VCAM-1 binding peptides were added on the nanoparticles by chemical bonding to form final PC-PLNs. The grafting rate is $71.24 \%$ (Section S1.4 in the ESM). PC-PLNs are spherical (Fig.3(c)), having an average size of $114.0 \pm 1.85 \mathrm{~nm}$, a polydispersity index (PDI) of $0.207 \pm 0.015$ and zeta potential of $11.86 \pm 0.95 \mathrm{mV}$ (Fig. $\mathrm{S} 1$ in the ESM). Encapsulation efficiency of CLT was $94.83 \% \pm$
$0.87 \%$ and loading capacity was $2.83 \% \pm 0.025 \%$. Moreover, PCPLNs exhibited excellent in vitro stability, displaying minor changes of particle size and PDI in buffer for a week at $4{ }^{\circ} \mathrm{C}$ (Figs. 3(e) and 3(f)) and for at least $24 \mathrm{~h}$ in serum (Fig. 3(d)). For experimental purposes, other PLNs including non-modified but positive charged C-PLNs and NC-PLNs were also generated (Section S1.1 and Table S1 in the ESM). Besides, all DiD-loaded PLNs were prepared as same as CLT-loaded PLNs, except that CLT was replaced by $\mathrm{DiD}$ (Section S1.2 in the ESM).

\subsection{PC-PLNs displayed better cellular uptake and kidney accumulation}

Uptake of different PLNs by LPS-activated cells was first assessed in vitro. As expected, the uptake of PD-PLNs was significantly increased compared with D-PLNs and other PLNs, suggesting that peptide modification could increase LPS-activated cells uptake (Figs. 4(a) and 4(b)). Additionally, the uptake of Neg-D-PLNs was significantly lower than all other groups, indicating that inflammatory cells would take up more positively charged particles as well. To further verify this conclusion, we pre-treated LPS-MPC5 and LPS-MRGEC with VCAM-1 antibody for $1 \mathrm{~h}$ before adding PD-PLNs (Figs. 4(c) and 4(d)). The results showed that antibodies pre-incubation indeed partially blocked the uptake of PD-PLNs by inflammatory cells. Hence, PD-PLNs could target LPS-activated cells in vitro and VCAM-1 played a role in enhancing PD-PLNs uptake by these cells. 


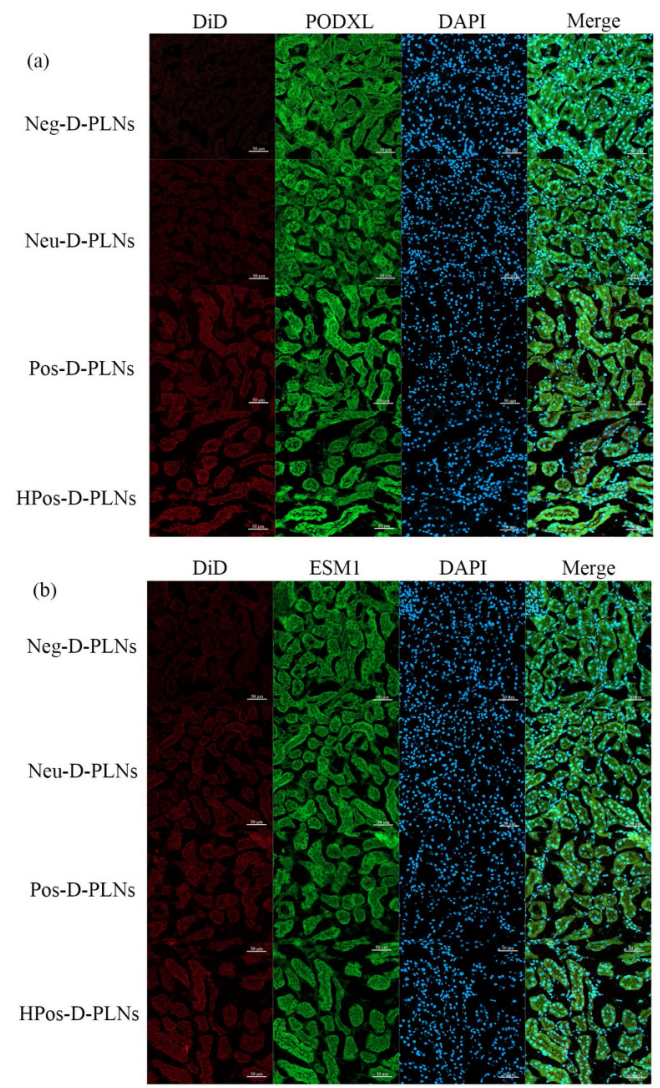

Figure 2 PLNs zeta-dependently accumulate in glomeruli cells. (a) Representative confocal images of D-PLNs localization at MPC5 cells, $n=3$. Scale bars, $50 \mu \mathrm{m}$. (b) Representative confocal images of D-PLNs localization at MRGEC cells, $n=3$. Scale bars, $50 \mu \mathrm{m}$.
We then investigated which pathway might be related to the PD-PLNs uptake by using LPS-MPC5 and LPS-MRGEC with inhibitors of different endocytic pathways (low temperature, Chol, nystatin, and dimethylacetamide (DMA)) (Figs. 4(e) and 4(f)). Unsurprisingly, low temperature $\left(4^{\circ} \mathrm{C}\right)$ inhibited the uptake of PDPLNs in both cell lines, indicating that the uptake is mainly mediated by active transport. It is noteworthy that nystatin exerted much stronger effect in MPC5 cells, while Chol worked more significantly in MRGEC cells. Thus, it appears that MPC5 relied heavily on caveolin-dependent pathway, and MRGEC mainly internalized nanoparticles via clathrin-mediated endocytosis. Besides, MPC5 also utilized macropinocytosis to internalize PDPLNs, which may explain its relatively independency on energy consumption.

Following in vitro experiments, we studied the distribution of PLNs in vivo. The main causes of CKD include different glomerulonephritis, diabetic nephropathy, hypertensive nephropathy, and tubular interstitial disease. Therefore, two animal models are suitable for CKD study: IgAN (a chronic glomerulonephritis) and CPN (a chronic progressive kidney disease with renal tubular damage) (Section S1.5 in the ESM). As both CPN and IgAN models provide inflammatory environment in kidney, we selected CPN model here as it is easier to establish. Here, different PLNs were given to modeled mice, and after $2 \mathrm{~h}$ their kidneys were removed and examined (Fig. 4(g)). In healthy mice, PD-PLNs showed similar accumulation in kidney with DPLNs, and both accumulate significantly more than ND-PLNs. In contrast, level of PD-PLNs in inflamed kidney was significantly increased while D-PLNs accumulation remained about the same. Therefore, the targeting ability of PD-PLNs is closely related to the surface modification and positive zeta potential, which is (a)

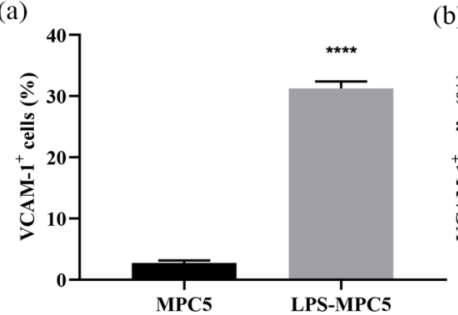

(c)

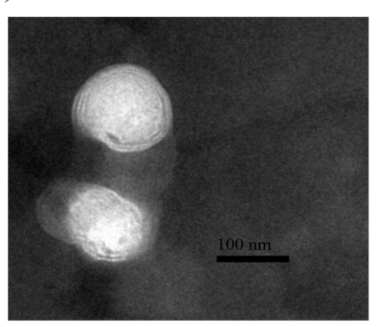

(e)

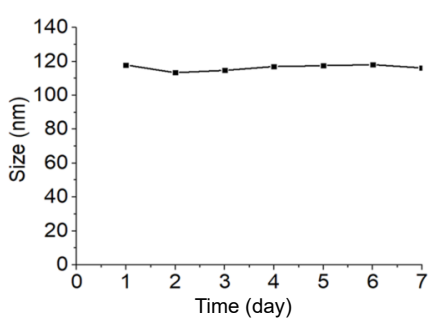

(b)

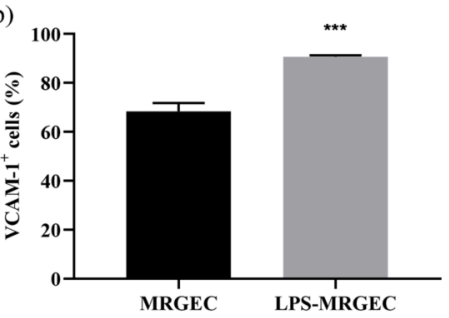

(d)

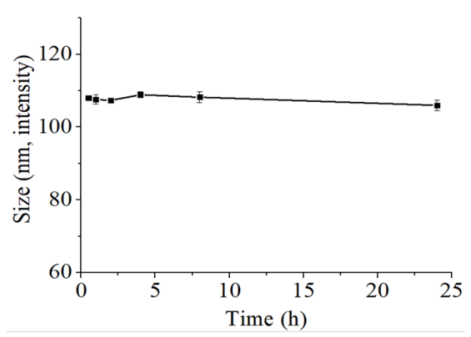

(f)

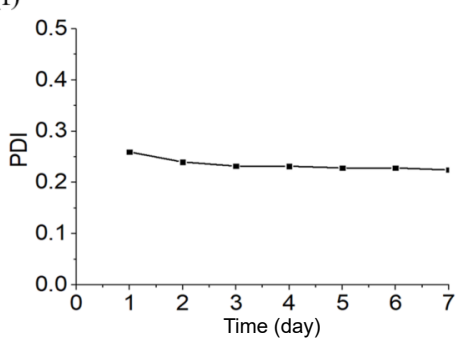

Figure 3 Construction of targeted drug delivery system. (a) and (b) Flow cytometric analyses of the VCAM-1 expression level on MPC5 (a) and MRGEC (b) activated by LPS or not. (c) Transmission electron micrographs of PC-PLNs. Scale bars, $100 \mathrm{~nm}$. (d) Stability assay of PC-PLNs in serum at $37^{\circ} \mathrm{C}$ indicated by change of size distribution for $24 \mathrm{~h}$. (e) and (f) Stability assay of PC-PLNs indicated by change of size distribution and PDI as a function of time in $4{ }^{\circ} \mathrm{C}$. All results are shown as mean $\pm \mathrm{SD}, n=3 .{ }^{* *} \mathrm{P}<0.001,{ }^{* * *} \mathrm{P}<0.0001$ vs. MPC5 group in Fig. 2(a), vs. MRGEC group in (b). 
(a)

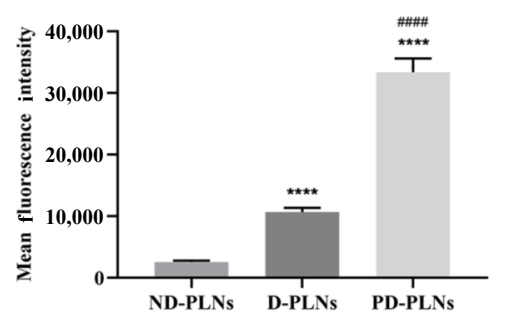

(b)

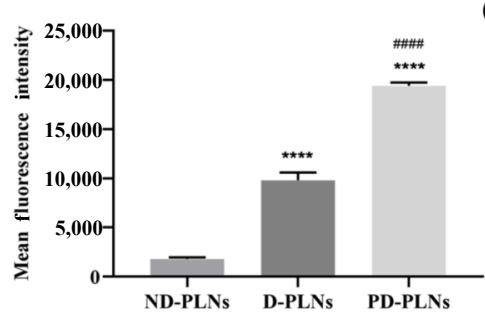

(c)

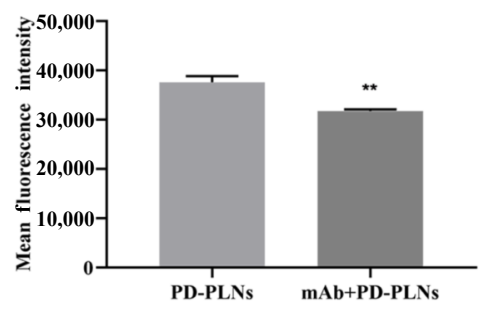

(d)

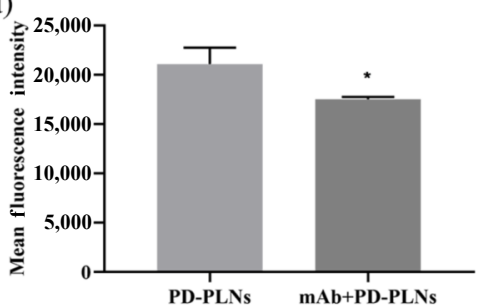

(e)

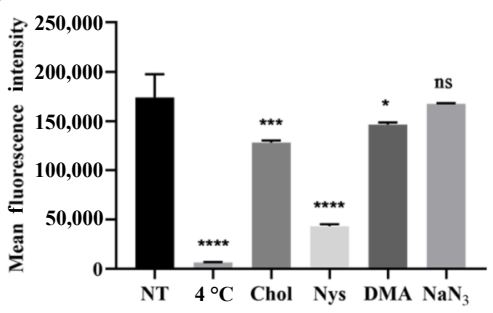

(g)

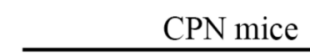

Healthy mice

(f)
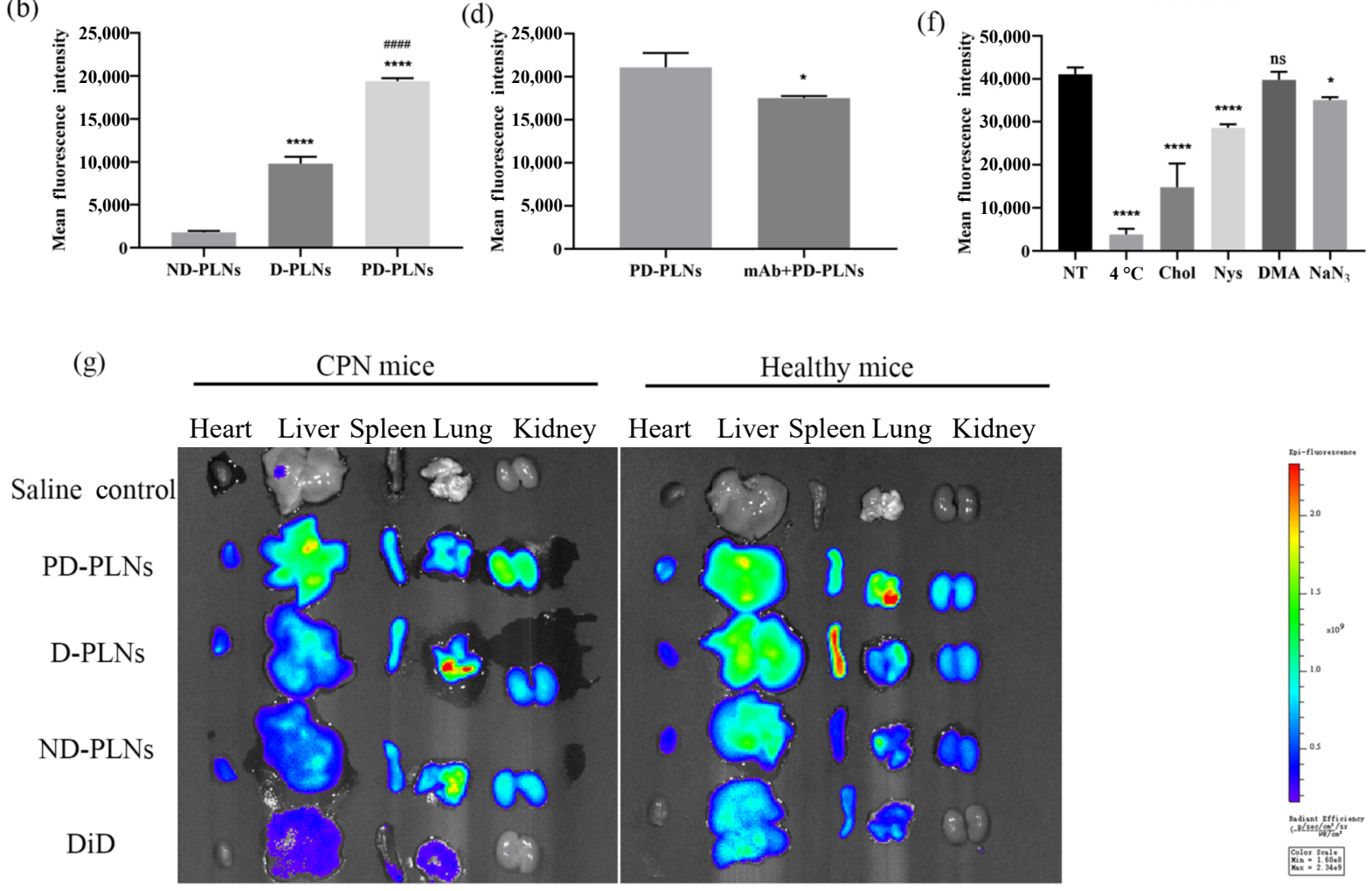

Figure 4 Peptides coupled PLNs successfully improve targeting in vitro and in vivo. (a) and (b) Flow cytometric analyses of uptake of different D-PLNs by LPS-MPC5 (a) and LPS-MRGEC (b), all results are shown as mean $\pm \mathrm{SD}, n=3$. ${ }^{* * * *} P<0.0001$ vs. ND-PLNs group, \#\#\#P<0.0001 vs. D-PLNs group. (c) and (d) Flow cytometric analyses of LPS-MPC5 (c) and LPS-MRGEC (d) treated with PD-PLNs for $4 \mathrm{~h}$ at $37^{\circ} \mathrm{C}$ with or without anti-VCAM-1 antibody pre-incubated, all results are shown as mean $\pm \mathrm{SD}, n=3 .{ }^{*} P<0.05$ vs. ND-PLNs group. (e) and (f) Effect of $4{ }^{\circ} \mathrm{C}$ and endocytic inhibitor on cellular uptake of PD-PLNs in LPS-MPC5 (e) and in LPS-MRGEC (f), all results are shown as mean $\pm \mathrm{SD}, n=3 .{ }^{*} P<0.05,{ }^{* * *} P<0.001,{ }^{* * *} P<0.0001$, ns, not significant vs. NT group. (g) Ex vivo imaging of organs harvested from mice at $2 \mathrm{~h}$ after administrated saline or DiD or D-PLNs.

consistent with the results of in vitro.

In summary, PD-PLNs displayed the highest uptake by inflammatory cells in vitro and accumulation in inflamed kidney in vivo, which is dependent on its surface charge and modification as proposed.

\subsection{PC-PLNs alleviated renal endothelium injury and inflammation in nephropathy mice}

Subsequently, we studied the therapeutic efficacy of PC-PLNs in vivo, using both IgAN and CPN animal models.

In IgAN mice, drugs were given every two days for a total of 4 doses, then the urine of mice was collected for analysis and the mice were sacrificed and examined (Fig. 5(a)). Compared to the blank group, PC-PLNs greatly reduced the urine protein level (Fig. 5(b)) and the levels of Cre and Urea (Figs. 5(c) and 5(d)). Tissue sections from modeled mice show clear abnormalities including high glomerular cells proliferation level, expanded mesangial matrix with widened mesangial area, epithelial swelling at renal tubules (accompanied by necrosis, shedding and casts), renal interstitial hyperemia and high inflammatory cells infiltration. Besides, IgA immunoglobulin depositions were also present in the mesangial region of the glomerulus. All these symptoms were substantially improved by PC-PLNs while other PLNs and free CLT displayed weaker effects (Figs. 5(e) and 5(f) and Figs. S2(a) and S2(b) in the ESM). These evidences indicate that CLT could alleviate renal injury and inflammation in IgAN mice, and PC-PLNs provided the best results.

As for CPN mice, cisplatin induced-CPN was characterized by chronic inflammation, interstitial fibrosis, development of glomerulosclerosis and endothelial dysfunction. After the same treatment regime as with IgAN model, the mice were again examined (Fig. 6(a)). PC-PLNs yet again displayed the strongest therapeutic effects. First, PC-PLNs returned the urine protein level (Fig. 6(b) and the levels of Cre and Urea closest back to normal (Figs. 6(e) and 6(f)). In CPN mice, clear damages in glomerulus were identifiable, such as loss of glomerulus volume, moderate to severe atrophied, swollen renal tubular epithelium (e.g., partial necrosis and vacuoles formation), diffusely thickened basement membrane, interstitial hyperemia, and infiltrated inflammatory- 
(a) Normal mice (NT)

IgAN mice

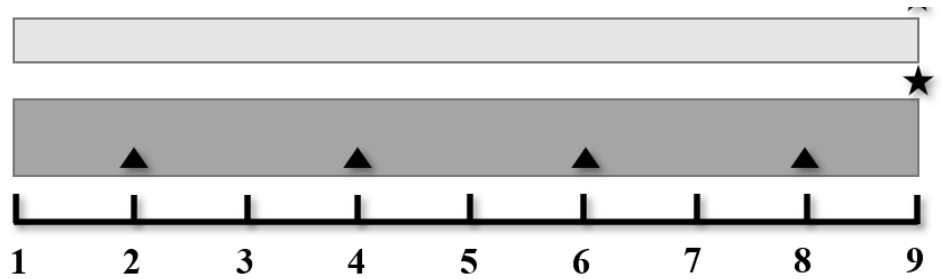

(b)

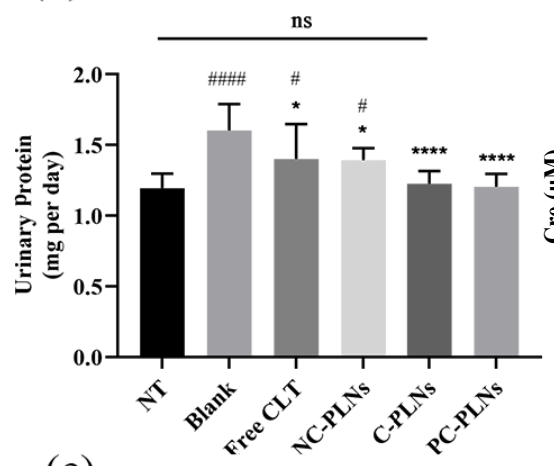

(e)

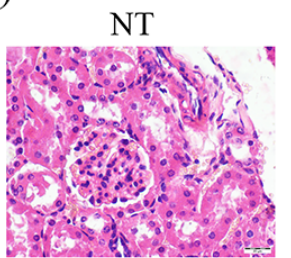

NC-PLNs

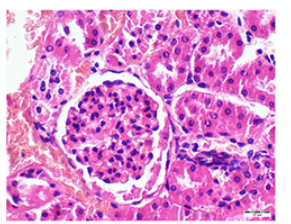

Blank

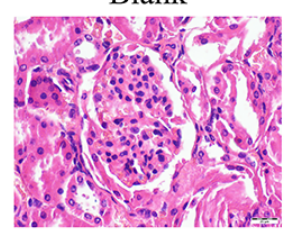

C-PLNs

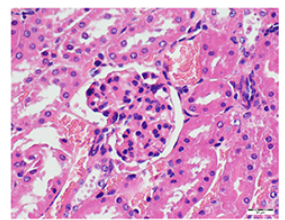

(c)

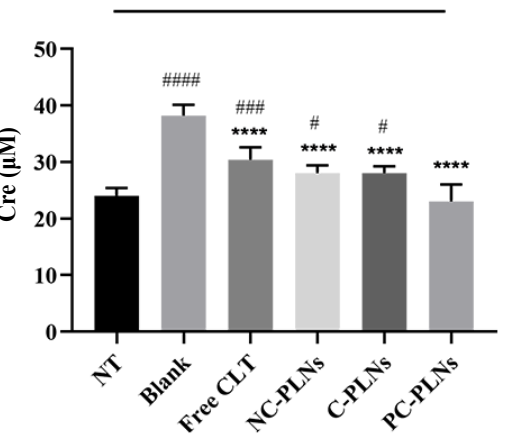

(d)

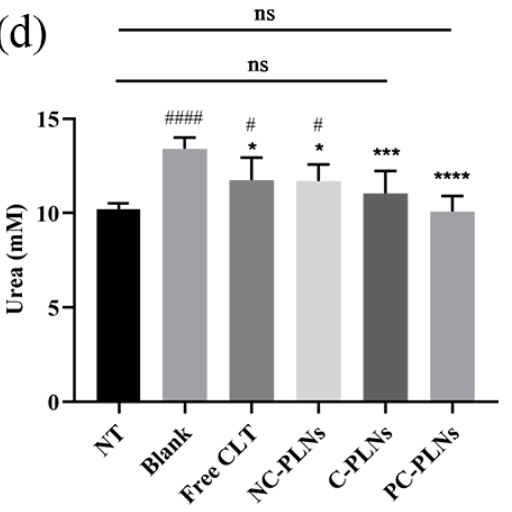

(f)

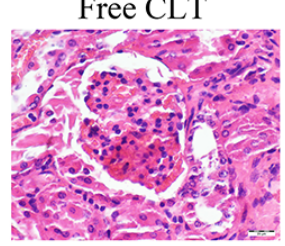

PC-PLNS
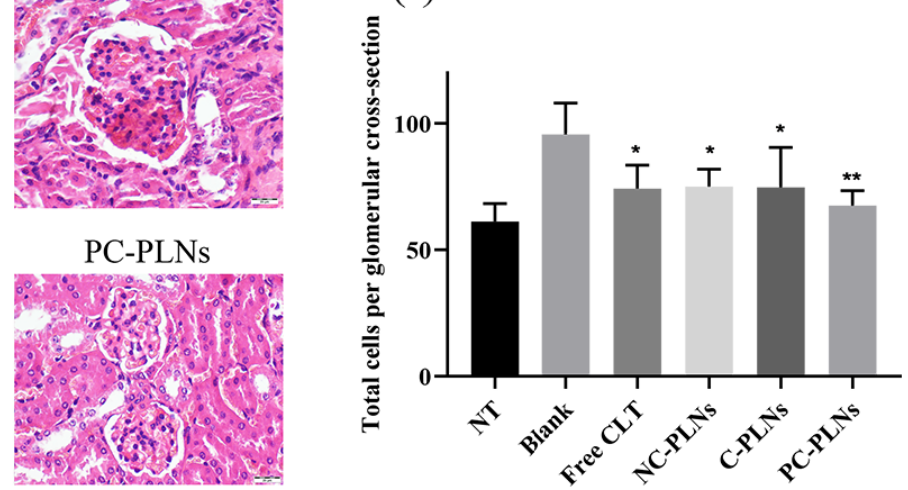

Figure 5 PC-PLNs alleviated renal endothelium injury and inflammation in IgAN mice $(n=5)$. (a) Schematic diagram of the treatment regime in IgAN mice. Black triangle denotes i.v. drug injections; black star denotes animal sacrifice and subsequent nephrectomy. A detailed description is given in the ESM. (b) 24-h urinary

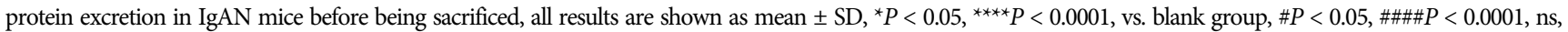
not significant vs. NT group. (c) and (d) The serum levels of Cre (c) and Urea (d) in IgAN mice on day 8 after treatment, all results are shown as mean \pm SD, ${ }^{\star} P<0.05$,

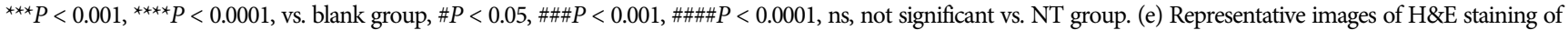
kidney sections. Scale bars, $20 \mu \mathrm{m}$. (f) Effects of treatment on total glomerular cellularity, all results are shown as mean $\pm \mathrm{SD},{ }^{\star} P<0.05,{ }^{* *} P<0.01$, vs. blank group.

related cells (Fig. 6(c)). PC-PLNs resulted the best damage recovery in glomerulus comparing with other treatments (Fig. 6(c)). In addition, PC-PLNs also outperformed all other treatment groups in preventing cell apoptosis (Section S1.6 in the ESM, Figs. 6(d) and 6(g)).

Besides, PAS staining (Figs. S3(a) and S4(a) in the ESM) and PCNA immunostaining (Figs. S3(b), S3(c), S4(b) and S4(c) in the ESM) were also presented to observe the efficacy of antiproliferation.

Therefore, PC-PLNs are effective for treating both IgAN and CPN mice. With relatively low CLT dose (in comparison to tumor treatment), PC-PLNs ameliorated glomerulus damage and reduced inflammation in kidney.

\subsection{Mechanic study of CLT on nephritis treatment}

In the above experiments, we demonstrated the therapeutic effect of CLT-loaded PC-PLNs, however, how exactly did it ameliorate kidney abnormality is still unclear. Following previous clues, we investigated the mechanism of CLT in the treatment of nephritis with a focus on kidney inflammation. In IgAN mice, the decrease of CD34 positive cells (Figs. 7(a) and 7(e)) and the increase of VCAM-1 expression (Figs. 7(b) and 7(f)) indicated that there is endothelial damage. Correspondingly, the expression of inflammation-related TNF- $\alpha$ was also significantly increased (Figs. 7(d) and 7(i)). After the administration of PC-PLNs, the number of $\mathrm{CD} 34$ positive cells in mice increased and the expression of VCAM- 1 and TNF- $\alpha$ were significantly reduced (Fig. 7). It was reported that TNF- $\alpha$ can upregulate VCAM-1 through the NF- $\kappa$ B pathway [30,41-43], but CLT could act as an inhibitor of NF- $\kappa B[9,12,15,16,36-39]$. Hence, it is possible that CLT inhibit the expression of downstream VCAM-1 via downregulating both TNF- $\alpha$ and NF- $\kappa$ B pathway. Considering the function of VCAM-1, this may reduce adhesion and aggregation of leukocytes to endothelial cells, hence ameliorate inflammation and endothelial cells damage.

CLT is also reported to regulate level of nitric oxide (NO)- an anti-inflammatory molecule with protective effect on endothelium which can delay nephritis progress $[28,41-49]$. With insufficient $\mathrm{NO}$, vasodilator function weakens in kidney and the possibility of 
(a)

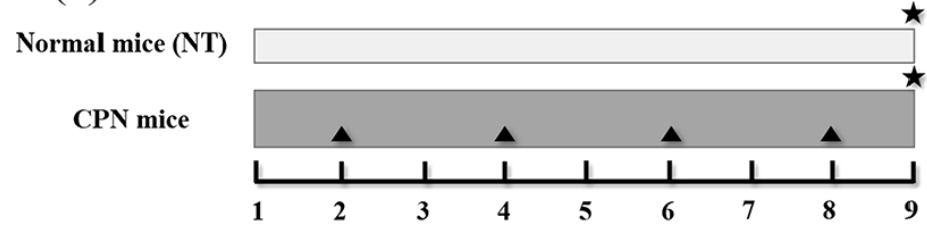

(c)

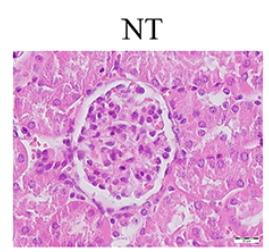

NC-PLNs

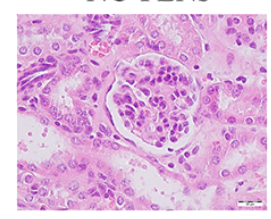

(e)

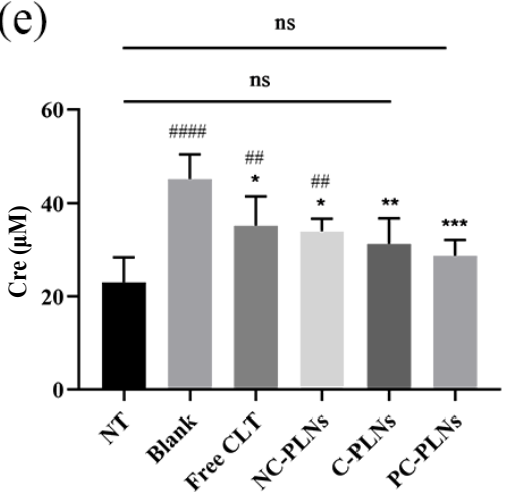

(d)
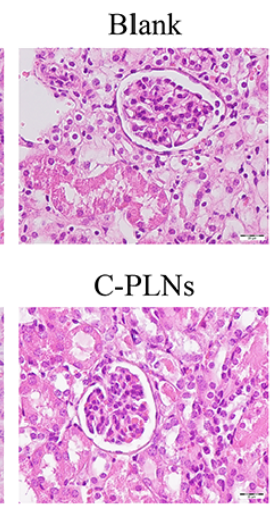

(f)

Free CLT

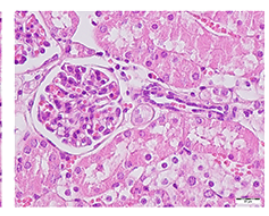

PC-PLNs
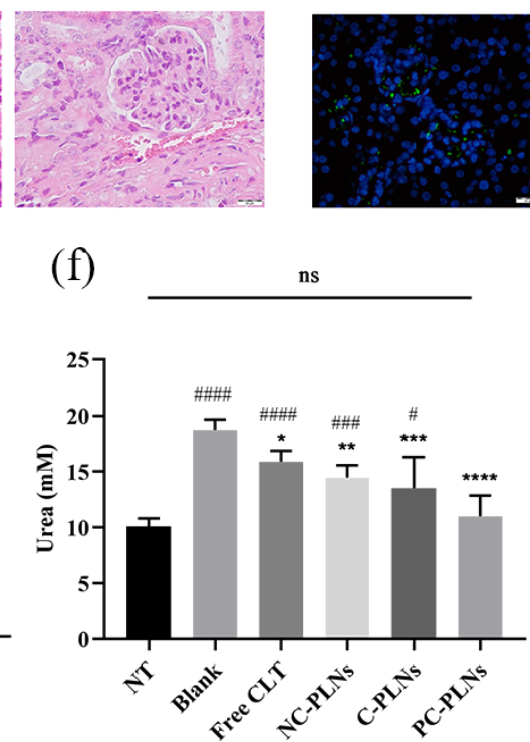

NT

NC-PLNs
C-PLNs (b)
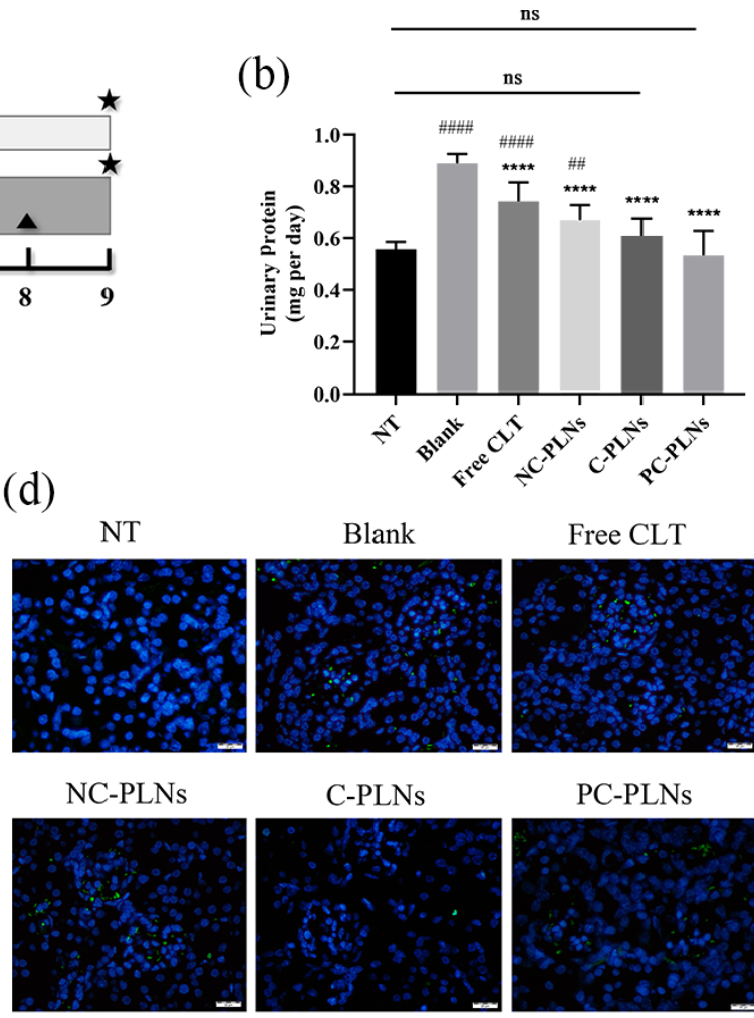

C-PLNs

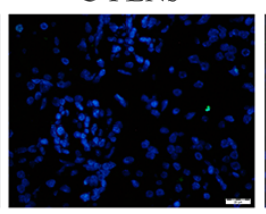

PC-PLNs

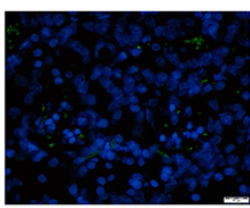

(g)

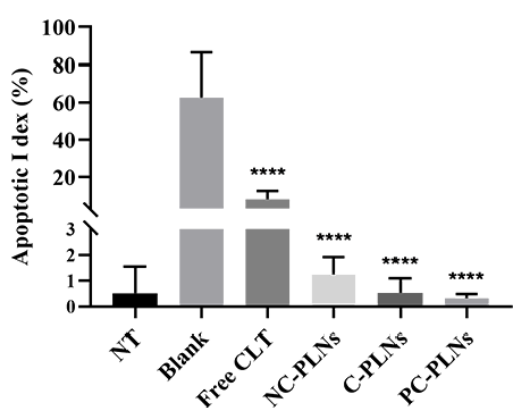

Figure 6 PC-PLNs alleviated renal injury and inflammation in CPN mice $(n=5)$. (a) Schematic diagram of the treatment regime in CPN mice. Black triangle denotes i.v. drug injections; black star denotes animal sacrifice and subsequent nephrectomy. A detailed description is given in the ESM. (b) 24-h urinary protein excretion in

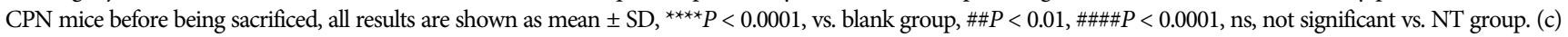
Representative images of H\&E staining of kidney sections. Scale bars, $20 \mu \mathrm{m}$. (d) Representative images of TUNEL-stained kidney tissues. Scale bars, $20 \mu \mathrm{m}$. (e)-(g) The serum levels of Cre (e) and Urea (f) in CPN mice on day 8 after treatment, all results are shown as mean $\pm \mathrm{SD},{ }^{\star} P<0.05,{ }^{* *} P<0.01$, ${ }^{\star * *} P<0.001,{ }^{\star * *} P<0.0001$, vs. blank group, $\# P<0.05$, \#\#P<0.01, \#\#\# <0.001, \#\#\#\#<0.0001, ns, not significant vs. NT group. (f) Representative images of TUNEL-stained kidney tissues. (g) Apoptotic index (AI) was semi-quantitatively based on TUNEL-stained results, all data are shown as mean $\pm \mathrm{SD},{ }^{* * * *} P<0.0001$ vs. blank group.

platelet aggregation increases; leukocytes may attach to glomerular endothelial cells more easily, impairing their permeability $[47,50]$. Here, we found that NO level indeed dramatically dropped in kidney of IgAN mice and PC-PLN (and other PLNs to a lesser extent) could increase its level back closer to normal (as in healthy kidney) (Fig. 7(h)). In fact, NO concentration is largely reversely correspondent to the pathological conditions of examined mice. Consistent phenomenon was identified in CPN mice too (Fig. S5 in the ESM). Previous reports have linked CLT with eNOS and it is known that TNF- $\alpha$ could inhibit eNOS expression [51-56]. Thus, CLT may rise the abnormally low NO level in kidney via downregulating the expression of TNF- $\alpha$ to strengthen the protective effect of $\mathrm{NO}$ and prevent further deterioration $[52,54$, $57,58]$.

In summary, CLT appears to be able to suppress inflammation and repair kidney tissue mainly via TNF- $\alpha$ downregulation to control level of downstream VCAM-1 and NO level, and NF- $\kappa B$ may also play a role in this process.

\subsection{PC-PLNs displayed low toxicity both in vitro and in} vivo

As CLT is toxic, a comprehensive safety evaluation was necessary to assess the toxicity of PC-PLNs. First, in vitro CCK-8 assays illustrated that PC-PLNs had lower cytotoxicity than free CLT (Figs. 8(a) and 8(b)). Then, we studied the in vivo toxicity of free CLT or PC-PLNs using mice. Analyzing the levels of AST, ALT, BILT, CKMB, LDH, Cre and Urea in the serum after different treatments with effective dosage, it is clear that free CLT displayed cardiac and renal toxicity while PC-PLNs was significantly less toxic (Fig. 8(c)). The organs of these mice were then removed and stained with H\&E and analyzed by a professional pathologist. Consistent with previous results, free CLT damaged heart and kidneys, but not the liver, spleen, and lungs; whereas PC-PLNs caused less damage in heart and kidney (Fig. 8(d)). 
(a)

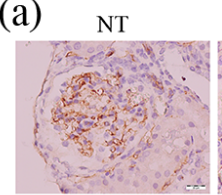

NC-PLNs

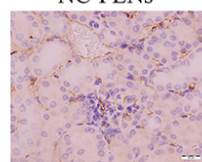

(b)

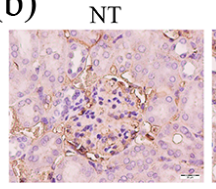

NC-PLNs

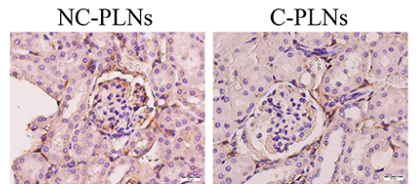

(c)

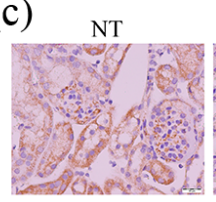

NC-PLNS

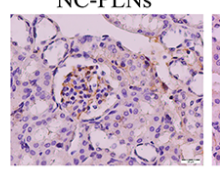

Blank

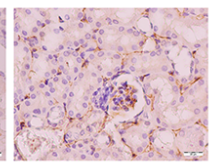

C-PLNs

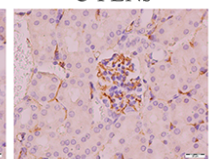

Blank

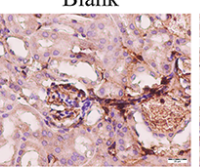

C-PLNs

Blank

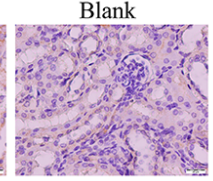

C-PLNs

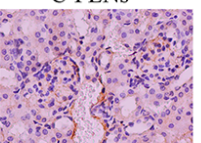

Free CLT

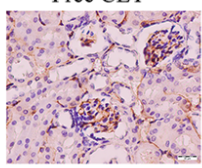

PC-PLNs

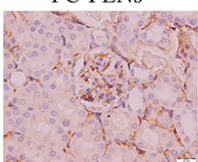

(d)
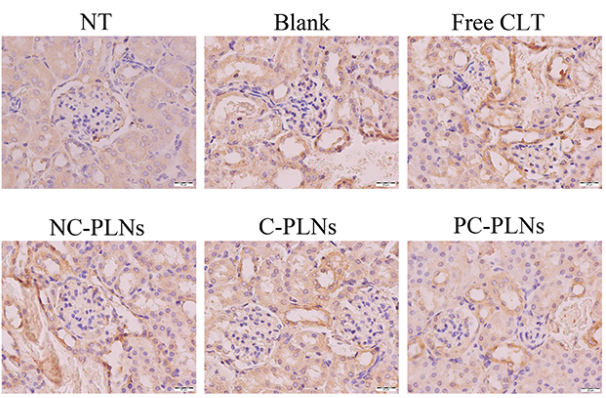

(e)

(f)

\section{政} PC-PLNS

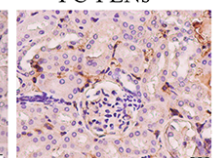

Free CLT

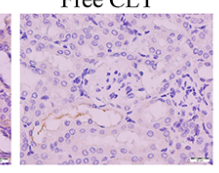

PC-PLNS

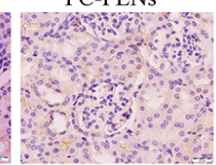

(g)
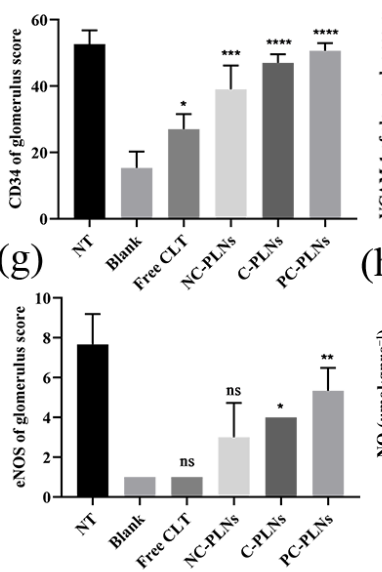

(h)
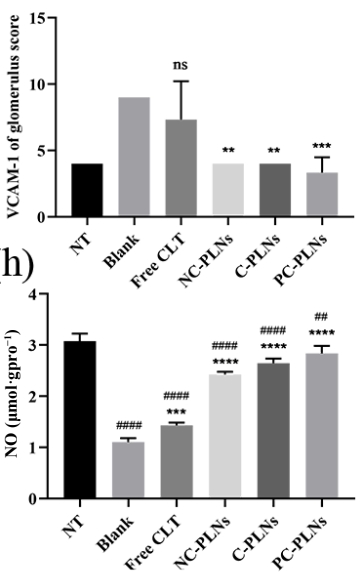

(i)

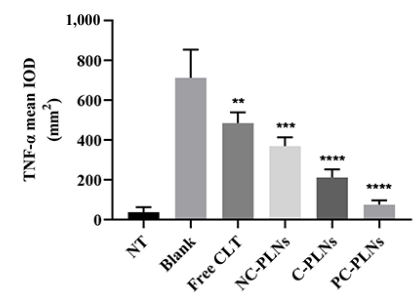

Figure 7 The possible mechanism of PC-PLNs in the treatment of nephritis $(n=5)$. (a) Representative images of CD34-stained kidney tissues. Scale bars, $20 \mu \mathrm{m}$. (b) Representative images of VCAM-1-stained kidney tissues. Scale bars, $20 \mu \mathrm{m}$. (c) Representative images of eNOS-stained kidney tissues. Scale bars, $20 \mu \mathrm{m}$. (d) Representative images of TNF- $\alpha$ - stained kidney tissues. Scale bars, $20 \mu \mathrm{m}$. (e) The levels of CD34 were semi-quantitatively scored, all data are shown as mean \pm SD, ${ }^{*} P$ $<0.05$, ${ }^{* *} P<0.001$, ${ }^{* * * *} P<0.0001$, vs. blank group. (f) The levels of VCAM-1 were semi-quantitatively scored, all data are shown as mean $\pm \mathrm{SD}$, ${ }^{* *} P<0.01$, ${ }^{* * *} P<$ 0.001 , ns, not significant vs. blank group. (g) The levels of eNOS were semi-quantitatively scored, all data are shown as mean $\pm \mathrm{SD}$, ${ }^{*} P<0.05$, ${ }^{* *} P<0.01$, ns, not significant vs. blank group. (h) The levels of renal NO, all data are shown as mean $\pm \mathrm{SD}$, ${ }^{* *} P<0.001,{ }^{* * *} P<0.0001$, vs. blank group, \#\#P<0.01, \#\#\#P< 0.0001 , vs. NT group. (i) The levels of TNF- $\alpha$ were quantitated, all data are shown as mean $\pm S D$, ${ }^{* *} P<0.01$, ${ }^{* * *} P<0.001$, ${ }^{* * *} P<0.0001$, vs. blank group.

\subsection{Discussion}

The etiology of CKD is complex and diverse, and there is no effective universal treatment method. Recently, some studies claimed that glomerular disease is the starting site of kidney disease $[1,2,19]$, hence treating unhealthy glomeruli may improve $\mathrm{CKD}$ caused by various etiologies. In addition, most delivery system to glomerulus is either not specifically targeting certain type of cells or targeting podocytes. We suggest that endothelial cells in glomerulus also play important roles during CKD. Therefore, we designed PC-PLNs for glomerulus endothelium targeting with the help of adequate size [11,22,24], optimized positive charge, and VCAM-1 binding peptide VHPKQHRGGSKGC. PC-PLNs exhibited excellent delivery enhancement and therapeutic effect as proposed, hence targeting glomerular endothelium is an effective strategy for CKD treatment.

During the study of CLT therapeutic mechanism, we found that CLT treatment raised kidney NO level. This seems to be contradictory to previous studies stating that CLT could inhibit the production of NO [8]. However, it is noteworthy that these reports worked on tumors and high dosage of CLT was applied for tumor treatment. Clearly, CKD and tumor are vastly different pathophysiological contexts. In fact, NO level is normally aberrantly high in tumor tissues, whereas most patients with CKD have relatively low levels of NO $[50,59,60]$. Therefore, CLT may exert different regulatory effects when used at different dosages and different microenvironments. In both cases, CLT helps to revert NO level back to normal, hence, it is possible that CLT affects an upstream regulation node that reacts to environment stimuli accordingly. Nevertheless, the detailed molecular mechanism of the link between CLT and NO requires further study.

\section{Conclusions}

In this study, a CLT-loaded delivery system (PC-PLN) with dual glomerulus targeting mechanisms was constructed for $\mathrm{CKD}$ treatment. PC-PLNs not only enriched in kidney, but also improved drug delivery directly into abnormal cells at glomerulus, especially to the endothelium. As far as we know, there are limited studies focusing on glomerular endothelial targeting. Our nanoparticles successfully reduced the toxicity of CLT, increased the accumulation of the target site, and enhanced the efficacy of CLT. Using PC-PLNs, we also investigated the molecular mechanism of CLT function in kidney. Our results indicated that 
(a)

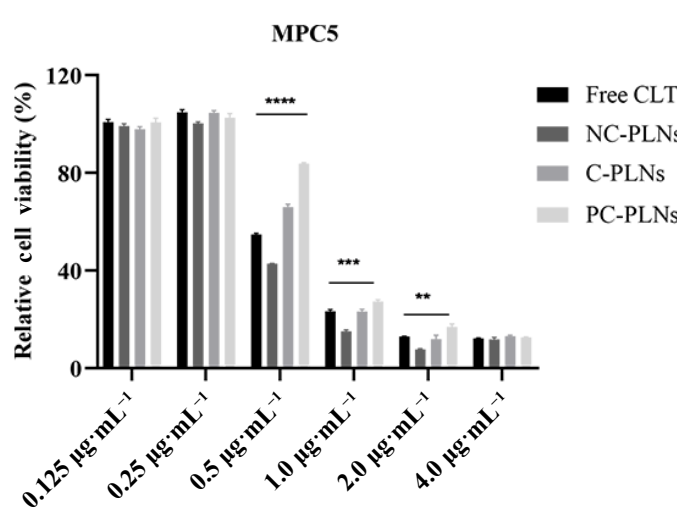

(c)
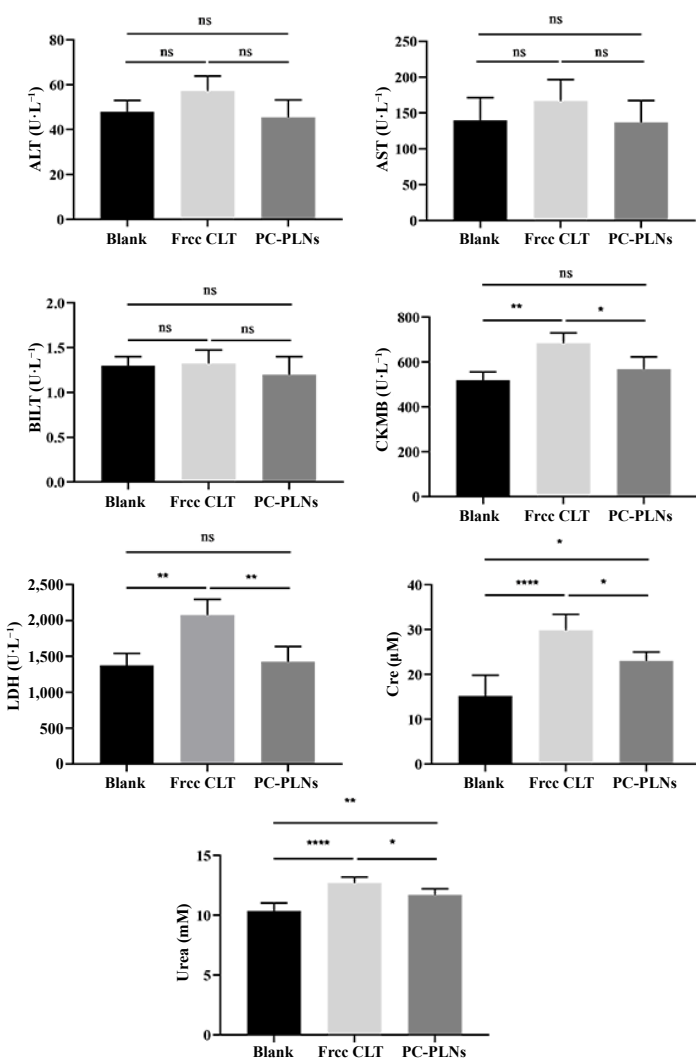

(b)

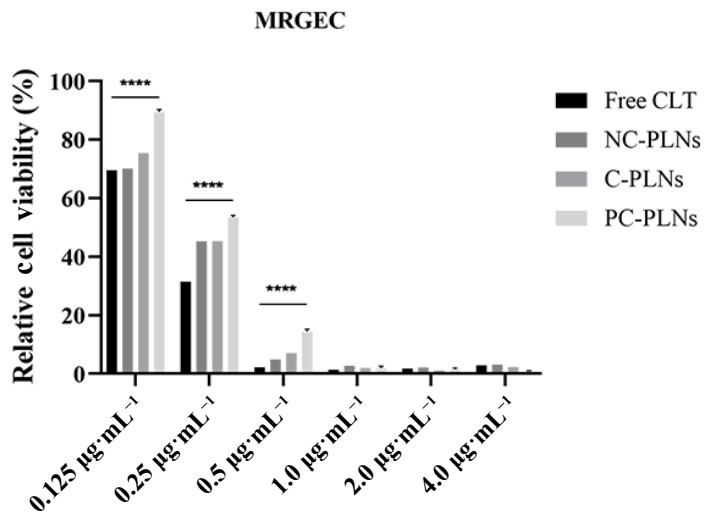

(d)
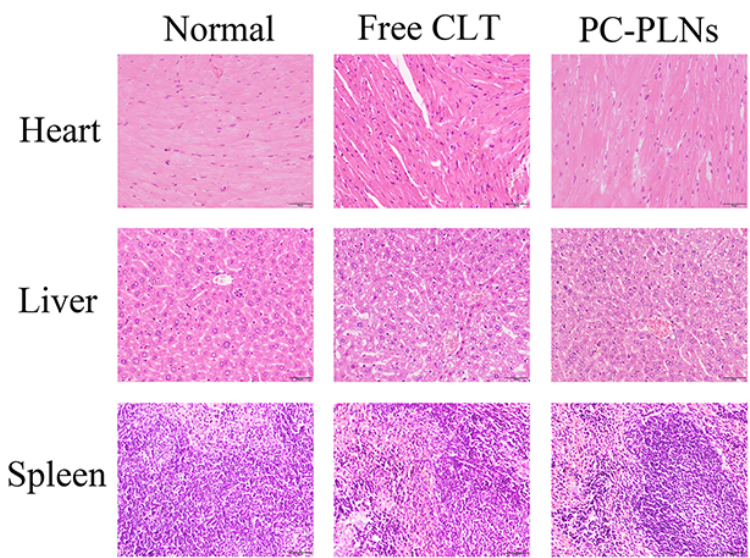

Lung
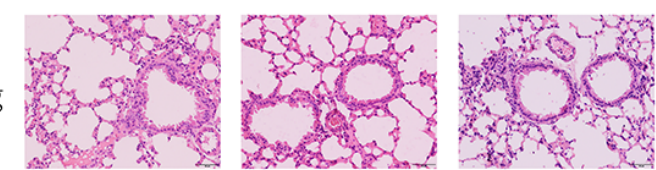

Kidney
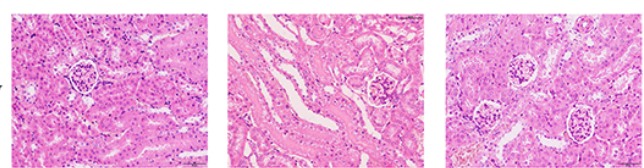

Figure 8 PC-PLNs displayed low toxicity both in vitro and in vivo. (a) and (b) CCK-8 assay of MPC5 (a) and MRGEC (b), all data are shown as mean \pm SD, $n=3$, ${ }^{* *} P<0.01,{ }^{* * *} P<0.001,{ }^{* * *} P<0.0001$, vs. free CLT group. (c) The levels of ALT, AST, BILT, CKMB, LDH, Cre and Urea in the serum, all data are shown as mean \pm SD, $n=5,{ }^{\star} P<0.05,{ }^{* *} P<0.01,{ }^{* * *} P<0.0001$, ns, not significant. (d) Representative images of H\&E staining assay of heart, liver, spleen, lung and kidney for healthy mice. Scale bars, $50 \mu \mathrm{m}$.

reducing glomerular injury can alleviate $\mathrm{CKD}$, and PC-PLNs offered clues for designing other glomerulus targeting delivery systems for CKD treatment.

\section{Acknowledgements}

This work was supported by the Regional Innovation and Development Joint Fund (No. U20A20411) and the National Science Fund for Excellent Young Scholars (No. 82022070).

Electronic Supplementary Material: Supplementary material (attached with all the supporting tables and figures mentioned in this work) is available in the online version of this article at https://doi.org/10.1007/s12274-021-3894-x.

\section{References}

[1] Lal, M. A.; Young, K. W.; Andag, U. Targeting the podocyte to treat glomerular kidney disease. Drug Discov. Today 2015, 20, $1228-1234$
[2] Liu, C. P.; Hu, Y.; Lin, J. C.; Fu, H. L.; Lim, L. Y.; Yuan, Z. X. Targeting strategies for drug delivery to the kidney: From renal glomeruli to tubules. Med. Res. Rev. 2019, 39, 561-578.

[3] Cascão, R.; Vidal, B.; Lopes, I. P.; Paisana, E.; Rino, J.; Moita, L. F.; Fonseca, J. E. Decrease of CD68 synovial macrophages in celastrol treated arthritic rats. PLoS One 2015, 10, e0142448.

[4] Liu, J. L.; Lee, J.; Hernandez, M. A. S.; Mazitschek, R.; Ozcan, U. Treatment of obesity with celastrol. Cell 2015, 161, 999-1011.

[5] Venkatesha, S. H.; Dudics, S.; Astry, B.; Moudgil, K. D. Control of autoimmune inflammation by celastrol, a natural triterpenoid. Pathog. Dis. 2016, 74, ftw059.

[6] Xie, G. S.; Zhu, L.; Song, Y. Y.; Huang, W.; Hu, D.; Cai, Z. W. An integrated quantitative proteomics strategy reveals the dual mechanisms of celastrol against acute inflammation. Chin. Chem. Lett. 2021, 32, 2164-2168.

[7] Cascão, R.; Fonseca, J. E.; Moita, L. F. Celastrol: A spectrum of treatment opportunities in chronic diseases. Front. Med. 2017, 4, 69.

[8] Gao, Y. F.; Zhou, S.; Pang, L. Z.; Yang, J. C.; Li, H. J.; Huo, X. W.; Qian, S. Y. Celastrol suppresses nitric oxide synthases and the angiogenesis pathway in colorectal cancer. Free Radic. Res. 2019, $53,324-334$ 
[9] Sha, M.; Ye, J.; Zhang, L.; Luan, Z.; Chen, Y.; Huang, J. Celastrol induces apoptosis of gastric cancer cells by miR-21 inhibiting PI3K/Akt-NF- $\kappa$ B signaling pathway. Pharmacology 2014, 93, 39-46.

[10] Venkatesha, S. H.; Moudgil, K. D. Celastrol and its role in controlling chronic diseases. Adv. Exp. Med. Biol. 2016, 928, 267-289.

[11] Guo, L.; Luo, S.; Du, Z. W.; Zhou, M. L.; Li, P. W.; Fu, Y.; Sun, X.; Huang, Y.; Zhang, Z. R. Targeted delivery of celastrol to mesangial cells is effective against mesangioproliferative glomerulonephritis. Nat. Commun. 2017, 8, 878.

[12] Kim, J. E.; Lee, M. H.; Nam, D. H.; Song, H. K.; Kang, Y. S.; Lee, J. E.; Kim, H. W.; Cha, J. J.; Hyun, Y. Y.; Han, S. Y. et al. Celastrol, an NF- $\kappa \mathrm{B}$ inhibitor, improves insulin resistance and attenuates renal injury in $\mathrm{db} / \mathrm{db}$ mice. PLoS One 2013, 8, e62068.

[13] Li, R.; Li, Y. P.; Zhang, J. H.; Liu, Q. H.; Wu, T.; Zhou, J.; Huang, H.; Tang, Q.; Huang, C. Y.; Huang, Y. et al. Targeted delivery of celastrol to renal interstitial myofibroblasts using fibronectin-binding liposomes attenuates renal fibrosis and reduces systemic toxicity. $J$. Control. Release 2020, 320, 32-44.

[14] Xu, S. H.; Feng, Y. Q.; He, W. S.; Xu, W.; Xu, W.; Yang, H. J.; Li, $\mathrm{X}$. Y. Celastrol in metabolic diseases: Progress and application prospects. Pharmacol. Res. 2021, 167, 105572.

[15] Zhao, Y.; Tan, Y. N.; Meng, T. T.; Liu, X.; Zhu, Y.; Hong, Y.; Yang, X. Q.; Yuan, H.; Huang, X.; Hu, F. Q. Simultaneous targeting therapy for lung metastasis and breast tumor by blocking the NF- $\kappa \mathrm{B}$ signaling pathway using celastrol-loaded micelles. Drug Deliv. 2018, $25,341-352$

[16] Zhou, L. L.; Lin, Z. X.; Fung, K. P.; Cheng, C. H. K.; Che, C. T.; Zhao, M.; Wu, S. H.; Zuo, Z. Celastrol-induced apoptosis in human $\mathrm{HaCaT}$ keratinocytes involves the inhibition of NF- $\kappa \mathrm{B}$ activity. Eur. J. Pharmacol. 2011, 670, 399-408.

[17] Li, L.; Liao, J. L.; Yuan, Q.; Hong, X.; Li, J.; Peng, Y. L.; He, M. Z.; Zhu, H. L.; Zhu, M. S.; Hou, F. F. et al. Fibrillin-1-enriched microenvironment drives endothelial injury and vascular rarefaction in chronic kidney disease. Sci. Adv. 2021, 7, eabc7170.

[18] Sharp, C. N.; Doll, M. A.; Megyesi, J.; Oropilla, G. B.; Beverly, L. J.; Siskind, L. J. Subclinical kidney injury induced by repeated cisplatin administration results in progressive chronic kidney disease. Am. J. Physiol. Renal Physiol. 2018, 315, F161-F172.

[19] Jourde-Chiche, N.; Fakhouri, F.; Dou, L.; Bellien, J.; Burtey, S.; Frimat, M.; Jarrot, P. A.; Kaplanski, G.; Le Quintrec, M.; Pernin, V. et al. Endothelium structure and function in kidney health and disease. Nat. Rev. Nephrol. 2019, 15, 87-108.

[20] Kitching, A. R.; Hutton, H. L. The players: Cells involved in glomerular disease. Clin. J. Am. Soc. Nephrol. 2016, 11, 1664-1674.

[21] Ma, Y. H.; Cai, F. H.; Li, Y. Y.; Chen, J. H.; Han, F.; Lin, W. Q. A review of the application of nanoparticles in the diagnosis and treatment of chronic kidney disease. Bioact. Mater. 2020, 5, 732-743.

[22] Wang, Y. F.; Wu, Q. S.; Wang, J. D.; Li, L.; Sun, X.; Zhang, Z. R.; Zhang, L. Co-delivery of p38 $\alpha$ MAPK and p65 siRNA by novel liposomal glomerulus-targeting nano carriers for effective immunoglobulin a nephropathy treatment. J. Control. Release 2020, $320,457-468$

[23] Xu, C.; Chang, A.; Hack, B. K.; Eadon, M. T.; Alper, S. L.; Cunningham, P. N. TNF-mediated damage to glomerular endothelium is an important determinant of acute kidney injury in sepsis. Kidney Int. 2014, 85, 72-81.

[24] Wang, G. W.; Li, Q. Y.; Chen, D. F.; Wu, B. H.; Wu, Y. L.; Tong, W. J.; Huang, P. T. Kidney-targeted rhein-loaded liponanoparticles for diabetic nephropathy therapy via size control and enhancement of renal cellular uptake. Theranostics 2019, 9, 6191-6208.

[25] Assady, S.; Wanner, N.; Skorecki, K. L.; Huber, T. B. New insights into podocyte biology in glomerular health and disease. J. Am. Soc. Nephrol. 2017, 28, 1707-1715.

[26] Lu, C. C.; Wang, G. H.; Lu, J.; Chen, P. P.; Zhang, Y.; Hu, Z. B.; Ma, K. L. Role of podocyte injury in glomerulosclerosis. Adv. Exp. Med. Biol. 2019, 1165, 195-232.

[27] Visweswaran, G. R. R.; Gholizadeh, S.; Ruiters, M. H. J.; Molema, G.; Kok, R. J.; Kamps, J. A. A. M. Targeting rapamycin to podocytes using a vascular cell adhesion molecule-1 (VCAM-1)harnessed SAINT-based lipid carrier system. PLoS One 2015, 10, e0138870.

[28] Sol, M.; Kamps, J. A. A. M.; van den Born, J.; van den Heuvel, M. C.; van der Vlag, J.; Krenning, G.; Hillebrands, J. L. Glomerular endothelial cells as instigators of glomerular sclerotic diseases. Front. Pharmacol. 2020, 11, 573557.

[29] Wu, X. Y.; Guo, R. Q.; Chen, P. L.; Wang, Q.; Cunningham, P. N. TNF induces caspase-dependent inflammation in renal endothelial cells through a Rho- and myosin light chain kinase-dependent mechanism. Am. J. Physiol. Renal Physiol. 2009, 297, F316-F326.

[30] Ailuno, G.; Baldassari, S.; Zuccari, G.; Schlich, M.; Caviglioli, G. Peptide-based nanosystems for vascular cell adhesion molecule-1 targeting: A real opportunity for therapeutic and diagnostic agents in inflammation associated disorders. J. Drug Deliv. Sci. Technol. 2020, 55, 101461.

[31] Zhong, F.; Mallipattu, S. K.; Estrada, C.; Menon, M.; Salem, F.; Jain, M. K.; Chen, H. Y.; Wang, Y. J.; Lee, K.; He, J. C. Reduced krüppel-like factor 2 aggravates glomerular endothelial cell injury and kidney disease in mice with unilateral nephrectomy. Am. J. Pathol. 2016, 186, 2021-2031.

[32] Liu, D.; Huang, Y.; Chen, B. J.; Zeng, J.; Guo, N.; Zhang, S. F.; Liu, L. X.; Xu, H.; Mo, X. M.; Li, W. M. Activation of mammalian target of rapamycin pathway confers adverse outcome in nonsmall cell lung carcinoma. Cancer 2011, 117, 3763-3773.

[33] He, C. B.; Hu, Y. P.; Yin, L. C.; Tang, C.; Yin, C. H. Effects of particle size and surface charge on cellular uptake and biodistribution of polymeric nanoparticles. Biomaterials 2010, 31, 3657-3666.

[34] Levchenko, T. S.; Rammohan, R.; Lukyanov, A. N.; Whiteman, K. R.; Torchilin, V. P. Liposome clearance in mice: The effect of a separate and combined presence of surface charge and polymer coating. Int. J. Pharm. 2002, 240, 95-102.

[35] Chen, D. F.; Han, S. P.; Zhu, Y. Q.; Hu, F.; Wei, Y. H.; Wang, G. W. Kidney-targeted drug delivery via rhein-loaded polyethyleneglycol$c o$-polycaprolactone- $c o$-polyethyleneimine nanoparticles for diabetic nephropathy therapy. Int. J. Nanomed. 2018, 13, 3507-3527.

[36] Haque, S.; Pouton, C. W.; McIntosh, M. P.; Ascher, D. B.; Keizer, D. W.; Whittaker, M. R.; Kaminskas, L. M. The impact of size and charge on the pulmonary pharmacokinetics and immunological response of the lungs to PLGA nanoparticles after intratracheal administration to rats. Nanomed. :Nanotechnol., Biol. Med. 2020, 30, 102291

[37] Kong, D. H.; Kim, Y. K.; Kim, M. R.; Jang, J. H.; Lee, S. Emerging roles of vascular cell adhesion molecule-1 (VCAM-1) in immunological disorders and cancer. Int. J. Mol. Sci. 2018, 19, 1057.

[38] Li, R. R.; Kowalski, P. S.; Morselt, H. W. M.; Schepel, I.; Jongman, R. M.; Aslan, A.; Ruiters, M. H. J.; Zijlstra, J. G.; Molema, G.; van Meurs, M. et al. Endothelium-targeted delivery of dexamethasone by anti-VCAM-1 SAINT-O-Somes in mouse endotoxemia. PLoS One 2018, 13, e0196976.

[39] Calin, M.; Stan, D.; Schlesinger, M.; Simion, V.; Deleanu, M.; Constantinescu, C. A.; Gan, A. M.; Pirvulescu, M. M.; Butoi, E.; Manduteanu, I. et al. VCAM-1 directed target-sensitive liposomes carrying CCR2 antagonists bind to activated endothelium and reduce adhesion and transmigration of monocytes. Eur. J. Pharm. Biopharm. 2015, 89, 18-29.

[40] Kelly, K. A.; Nahrendorf, M.; Yu, A. M.; Reynolds, F.; Weissleder, R. In vivo phage display selection yields atherosclerotic plaque targeted peptides for imaging. Mol. Imaging Biol. 2006, 8, 201.

[41] Tousoulis, D.; Kampoli, A. M.; Papageorgiou, C. T. N.; Stefanadis, C. The role of nitric oxide on endothelial function. Curr. Vasc. Pharmacol. 2012, 10, 4-18.

[42] Verma, S. K.; Molitoris, B. A. Renal endothelial injury and microvascular dysfunction in acute kidney injury. Semin. Nephrol. 2015, 35, 96-107.

[43] Whiting, C.; Castillo, A.; Haque, M. Z.; Majid, D. S. A. Protective role of the endothelial isoform of nitric oxide synthase in ANG IIinduced inflammatory responses in the kidney. Am. J. Physiol. Renal Physiol. 2013, 305, F1031-F1041.

[44] Adler, S.; Huang, H.; Loke, K. E.; Xu, X. B.; Tada, H.; Laumas, A.; Hintze, T. H. Endothelial nitric oxide synthase plays an essential role 
in regulation of renal oxygen consumption by NO. Am. J. Physiol. Renal Physiol. 2001, 280, F838-F843.

[45] Baylis, C. Nitric oxide deficiency in chronic kidney disease. Am. J. Physiol. Renal Physiol. 2008, 294, F1-F9.

[46] Coleman, J. W. Nitric oxide: A regulator of mast cell activation and mast cell-mediated inflammation. Clin. Exp. Immunol. 2002, 129, 4-10.

[47] Dolinina, J.; Sverrisson, K.; Rippe, A.; Öberg, C. M.; Rippe, B. Nitric oxide synthase inhibition causes acute increases in glomerular permeability in vivo, dependent upon reactive oxygen species. Am. J. Physiol. Renal Physiol. 2016, 311, F984-F990.

[48] Fiore, M. C.; Jimenez, P. M.; Cremonezzi, D.; Juncos, L. I.; García, N. H. Statins reverse renal inflammation and endothelial dysfunction induced by chronic high salt intake. Am. J. Physiol. Renal Physiol. 2011, 301, F263-F270.

[49] Liu, B.; Xu, L. L.; Yu, X. M.; Li, W.; Sun, X. Z.; Xiao, S.; Guo, M. J.; Wang, H. F. Protective effect of KLF15 on vascular endothelial dysfunction induced by TNF- $\alpha$. Mol. Med. Rep. 2018, 18, 1987-1994.

[50] Roumeliotis, S.; Mallamaci, F.; Zoccali, C. Endothelial dysfunction in chronic kidney disease, from biology to clinical outcomes: A 2020 update. J. Clin. Med. 2020, 9, 2359.

[51] Guo, Y. M.; Li, P. F.; Gao, L.; Zhang, J. M.; Yang, Z. R.; Bledsoe, G.; Chang, E.; Chao, L.; Chao, J. L. Kallistatin reduces vascular senescence and aging by regulating microRNA-34a-SIRT1 pathway. Aging Cell 2017, 16, 837-846.

[52] Kim, J.; Lee, K. S.; Kim, J. H.; Lee, D. K.; Park, M.; Choi, S.; Park, W.; Kim, S.; Choi, Y. K.; Hwang, J. Y. et al. Aspirin prevents TNF$\alpha$-induced endothelial cell dysfunction by regulating the NF- $\kappa \mathrm{B}$ dependent miR-155/eNOS pathway: Role of a miR-155/eNOS axis in preeclampsia. Free Radic. Biol. Med. 2017, 104, 185-198.

[53] Valerio, A.; Cardile, A.; Cozzi, V.; Bracale, R.; Tedesco, L.;
Pisconti, A.; Palomba, L.; Cantoni, O.; Clementi, E.; Moncada, S. et al. TNF- $\alpha$ downregulates eNOS expression and mitochondrial biogenesis in fat and muscle of obese rodents. J. Clin. Invest. 2006, 116, 2791-2798.

[54] Choi, S.; Kim, J.; Kim, J. H.; Lee, D. K.; Park, W.; Park, M.; Kim, S.; Hwang, J. Y.; Won, M. H.; Choi, Y. K. et al. Carbon monoxide prevents TNF- $\alpha$-induced eNOS downregulation by inhibiting NF- $\kappa$ Bresponsive miR-155-5p biogenesis. Exp. Mol. Med. 2017, 49, e403.

[55] Zhang, J. D.; Patel, M. B.; Griffiths, R.; Mao, A.; Song, Y. S.; Karlovich, N. S.; Sparks, M. A.; Jin, H. X.; Wu, M.; Lin, E. E. et al. Tumor necrosis factor- $\alpha$ produced in the kidney contributes to angiotensin II-dependent hypertension. Hypertension 2014, 64, $1275-1281$.

[56] Liu, X.; Gao, R. W.; Li, M.; Si, C. F.; He, Y. P.; Wang, M.; Yang, Y.; Zheng, Q. Y.; Wang, C. Y. The ROS derived mitochondrial respirstion not from NADPH oxidase plays key role in celastrol against angiotensin II-mediated HepG2 cell proliferation. Apoptosis 2016, 21, 1315-1326.

[57] Matavelli, L. C.; Huang, J. Q.; Siragy, H. M. Angiotensin $\mathrm{AT}_{2}$ receptor stimulation inhibits early renal inflammation in renovascular hypertension. Hypertension 2011, 57, 308-313.

[58] Pandey, A.; Goru, S. K.; Kadakol, A.; Malek, V.; Gaikwad, A. B. Differential regulation of angiotensin converting enzyme 2 and nuclear factor- $\kappa \mathrm{B}$ by angiotensin II receptor subtypes in type 2 diabetic kidney. Biochimie 2015, 118, 71-81.

[59] Kruse, N. T. Nutraceuticals as a potential adjunct therapy toward improving vascular health in CKD. Am. J. Physiol. Regul., Integr. Comp. Physiol. 2019, 317, R719-R732.

[60] Pushpakumar, S.; Kundu, S.; Sen, U. Hydrogen sulfide protects hyperhomocysteinemia-induced renal damage by modulation of caveolin and eNOS interaction. Sci. Rep. 2019, 9, 2223. 